\title{
ASYMPTOTICALLY LINEAR PROBLEMS DRIVEN BY FRACTIONAL LAPLACIAN OPERATORS
}

\author{
ALESSIO FISCELLA, RAFFAELLA SERVADEI, AND ENRICO VALDINOCI
}

\begin{abstract}
In this paper we study a non-local fractional Laplace equation, depending on a parameter $\lambda$, with asymptotically linear right-hand side. Our main result concerns the existence of weak solutions for this equation and it is obtained using variational and topological methods. Namely, our existence theorem follows as an application of the Saddle Point Theorem. It extends some results, well known for the Laplace operator, to the nonlocal fractional setting.
\end{abstract}

\section{CONTENTS}

1. Introduction

2. The functional analytic setting $\quad 5$

3. Variational formulation of the problem 6

4. The non-resonant case 8

4.1. The case $\lambda<\lambda_{1}$

4.2. Proof of Theorem 1 in the non-resonant case, when $\lambda<\lambda_{1} \quad 8$

4.3. The case $\lambda>\lambda_{1}$

4.4. Proof of Theorem 1 in the non-resonant case, when $\lambda>\lambda_{1}$

5. The resonant case 13

5.1. Proof of Theorem 1 in the resonant setting 21

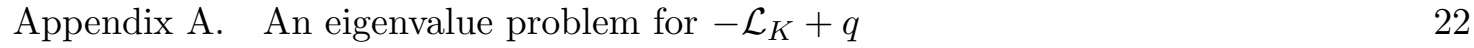

References $\quad 23$

\section{INTRODUCTION}

In the literature many papers are devoted to the study of non-local fractional Laplacian equations with superlinear and subcritical or critical growth (see $[2,4,5,8,13,14,16$, $17,18,19,20,22]$ and references therein). After studying this kind of problems in the recent papers cited above, here we deal with non-local equations with asymptotically linear right-hand side.

In the standard case of the Laplacian there is a wide literature on this topic (see, for instance, [1] and references therein). Aim of the present work is to provide some existence results for the non-local counterpart of such a problem, that is the equation

$$
\begin{cases}-\mathcal{L}_{K} u+q(x) u=\lambda u+f(u)+h(x) & \text { in } \Omega \\ u=0 & \text { in } \mathbb{R}^{n} \backslash \Omega,\end{cases}
$$

Key words and phrases. integrodifferential operators, fractional Laplacian, variational techniques, Saddle Point Theorem, Palais-Smale condition.

2010 AMS Subject Classification: Primary: 49J35, 35A15, 35S15; Secondary: 47G20, 45 G05.

The second author was supported by the MIUR National Research Project Variational and Topological Methods in the Study of Nonlinear Phenomena and the GNAMPA Project Variational Methods for the Study of Nonlocal Elliptic Equations with Fractional Laplacian Operators, while the third one by the MIUR National Research Project Nonlinear Elliptic Problems in the Study of Vortices and Related Topics and the FIRB Project A\&B (Analysis and Beyond). All the authors were supported by the ERC Grant $\epsilon$ (Elliptic Pde's and Symmetry of Interfaces and Layers for Odd Nonlinearities). 
where $s \in(0,1)$ is fixed, $n>2 s, \Omega \subset \mathbb{R}^{n}$ is an open, bounded set with Lipschitz boundary, $\lambda$ is a real parameter, $f, q$ and $h$ are sufficiently smooth functions and $\mathcal{L}_{K}$ is a general non-local operator defined as follows:

$$
\mathcal{L}_{K} u(x)=\int_{\mathbb{R}^{n}}(u(x+y)+u(x-y)-2 u(x)) K(y) d y,
$$

for all $x \in \mathbb{R}^{n}$. Here, the kernel $K: \mathbb{R}^{n} \backslash\{0\} \rightarrow(0,+\infty)$ is a function with the properties that

$$
m K \in L^{1}\left(\mathbb{R}^{n}\right) \text {, where } m(x)=\min \left\{|x|^{2}, 1\right\} ;
$$

there exists $\theta>0$ such that $K(x) \geqslant \theta|x|^{-(n+2 s)}$ for any $x \in \mathbb{R}^{n} \backslash\{0\}$;

$$
K(x)=K(-x) \text { for any } x \in \mathbb{R}^{n} \backslash\{0\} .
$$

A typical example for $K$ is given by $K(x)=|x|^{-(n+2 s)}$. In this case $\mathcal{L}_{K}=-(-\Delta)^{s}$ and problem (1.1) becomes

$$
\begin{cases}(-\Delta)^{s} u+q(x) u=\lambda u+f(u)+h(x) & \text { in } \Omega \\ u=0 & \text { in } \mathbb{R}^{n} \backslash \Omega,\end{cases}
$$

where $-(-\Delta)^{s}$ is the fractional Laplace operator which (up to normalization factors) may be defined as

$$
-(-\Delta)^{s} u(x)=\int_{\mathbb{R}^{n}} \frac{u(x+y)+u(x-y)-2 u(x)}{|y|^{n+2 s}} d y
$$

for $x \in \mathbb{R}^{n}$ (see [7] and references therein for further details on the fractional Laplacian).

Along the paper, we suppose that in equation (1.1) the function $f: \mathbb{R} \rightarrow \mathbb{R}$ verifies the following assumptions:

$$
f \in C^{1}(\mathbb{R})
$$

there exists a constant $M>0$ such that $|f(t)| \leqslant M$ for any $t \in \mathbb{R}$,

while $q, h: \Omega \rightarrow \mathbb{R}$ are such that

$$
q \in L^{\infty}(\Omega), q(x) \geqslant 0 \text { a.e. } x \in \Omega
$$

and

$$
h \in L^{2}(\Omega)
$$

respectively.

When $f \equiv 0$ and $h \equiv 0$ problem (1.1) becomes the following eigenvalue problem

$$
\begin{cases}-\mathcal{L}_{K} u+q(x) u=\lambda u & \text { in } \Omega \\ u=0 & \text { in } \mathbb{R}^{n} \backslash \Omega .\end{cases}
$$

We recall that there exists a non-decreasing sequence of positive eigenvalues $\lambda_{k}$ for which (1.12) admits a solution. We will study problem (1.12) in Appendix A, since, in the following, we need some information on the eigenvalues and the eigenfunctions of $-\mathcal{L}_{K}+q$.

Along the paper we consider both the resonant and the non-resonant case, that is the case when $\lambda$ belongs to the spectrum of the operator driving the equation and the one when $\lambda$ does not, respectively. As for the resonant setting we would like to note that we are able to treat this case only if $\lambda$ satisfies the following assumption (for more details see Section 5)

$\lambda$ is an eigenvalue of problem (1.12) such that

all the eigenfunctions corresponding to $\lambda$

have nodal set with zero Lebesgue measure.

As usual, the "nodal set" of a function $e$ in $\Omega$ is the level set $\{x \in \Omega: e(x)=0\}$.

Condition (1.13) is true, for instance, in the case of the fractional Laplace operator $(-\Delta)^{s}$, when $\lambda$ is its first eigenvalue, since the first eigenfunction of $(-\Delta)^{s}$ is strictly positive (see 
[17, Proposition 9 and Appendix A] and [21]). We also would like to note that this condition is compatible with the classical case of the Laplace operator (which corresponds to the choice $s=1$ ), since in this context it is satisfied by every eigenvalue (see e.g. [6, 10]).

We think that it is a very intriguing question to establish whether or not all the eigenfunctions of problem (1.12) have their nodal sets of vanishing measure, as it happens in the classical case of the Laplacian (i.e., to decide whether or not (1.13) is always satisfied). The answer to this question clearly goes far beyond the scope of this paper, since it must rely either on techniques that do not seem to have a simple counterpart for the fractional Laplacian (such as the Carleson-type estimates or the monotonicity formulas in the variables where the fractional Laplacian is computed) or on big results that are still unknown in this framework (for instance a Unique Continuation Principle for the fractional Laplace equations).

Also, in the resonant case, in order to prove our existence result, we need some extra conditions on the terms $f$ and $h$. Precisely, denoting by

$$
f_{l}=\lim _{t \rightarrow-\infty} f(t) \quad \text { and } \quad f_{r}=\lim _{t \rightarrow+\infty} f(t),
$$

we assume that

$$
f_{l} \text { and } f_{r} \text { exist, are finite and such that } f_{l}>f_{r}
$$

and

$$
\begin{array}{r}
f_{r} \int_{\Omega} \varphi^{-}(x) d x-f_{l} \int_{\Omega} \varphi^{+}(x) d x<\int_{\Omega} h(x) \varphi(x) d x<f_{l} \int_{\Omega} \varphi^{-}(x) d x-f_{r} \int_{\Omega} \varphi^{+}(x) d x \\
\text { for any } \varphi \in E_{\lambda} \backslash\{0\},
\end{array}
$$

where $\varphi^{+}=\max \{\varphi, 0\}$ and $\varphi^{-}=\max \{-\varphi, 0\}$ denote the positive and the negative part of the function $\varphi$, respectively, while $E_{\lambda}$ is the linear space generated by the eigenfunctions related to $\lambda$ (for a precise definition of $E_{\lambda}$ we refer to Section 5 ).

We would remark that these extra conditions on $f$ and $h$ are exactly the same required in the resonant setting, when dealing with the classical Laplace operator (see $[1$, Section 4.4.3]). Moreover, we would point out that in (1.14) the limits $f_{l}$ and $f_{r}$ have to be different, but the case $f_{l}<f_{r}$ would work as well, with some modifications in the main arguments. Assumption (1.15) is the classical Landesman-Lazer condition, firstly introduced in [11], which represents one of the natural sufficient condition ${ }^{1}$ given in order to obtain an existence result in a resonant setting.

As a model for $f$ we can take the function

$$
f(t)= \begin{cases}\frac{1}{1+t^{2}} & \text { if } t \geqslant 0 \\ 1 & \text { if } t<0 .\end{cases}
$$

\footnotetext{
${ }^{1}$ We point out that condition (1.15) is satisfied by every measurable function $h$ which ranges in $\left(-f_{l},-f_{r}\right)$. Indeed, in this case,

$$
f_{r}<-h(x)<f_{l},
$$

hence, multiplying by $\varphi^{ \pm}(x) \geqslant 0$ and integrating over $\Omega$

$$
f_{r} \int_{\Omega} \varphi^{ \pm}(x) d x<-\int_{\Omega} h(x) \varphi^{ \pm}(x) d x<f_{l} \int_{\Omega} \varphi^{ \pm}(x) d x .
$$

As a consequence

$$
\begin{aligned}
& f_{r} \int_{\Omega} \varphi^{-}(x) d x-f_{l} \int_{\Omega} \varphi^{+}(x) d x<-\int_{\Omega} h(x) \varphi^{-}(x) d x+\int_{\Omega} h(x) \varphi^{+}(x) d x \\
& \quad=\int_{\Omega} h(x) \varphi(x) d x=\int_{\Omega} h(x) \varphi^{+}(x) d x-\int_{\Omega} h(x) \varphi^{-}(x) d x \\
& \quad<-f_{r} \int_{\Omega} \varphi^{+}(x) d x+f_{l} \int_{\Omega} \varphi^{-}(x) d x
\end{aligned}
$$
}

that is (1.15). 
We would like to note that, in this case, $f$ does not satisfy the assumptions required in [9, Theorem 1], where an asymptotically linear problem at resonance driven by a general non-local operator was considered. Indeed, in [9] the asymptotically linear case when the primitive of $f$ goes to infinity was considered.

The main result of the present paper concerns the existence of weak solutions for problem (1.1). For this, first of all, we have to write the weak formulation of the problem. To this purpose, the fractional Sobolev space $H^{s}\left(\mathbb{R}^{n}\right)$ is not enough. This is the reason why we work in the spaces $X$ and $X_{0}$, introduced in [15] (see also $[16,17]$ for further properties).

The functional space $X$ denotes the linear space of Lebesgue measurable functions from $\mathbb{R}^{n}$ to $\mathbb{R}$ such that the restriction to $\Omega$ of any function $g$ in $X$ belongs to $L^{2}(\Omega)$ and

the map $(x, y) \mapsto(g(x)-g(y)) \sqrt{K(x-y)}$ is in $L^{2}\left(\left(\mathbb{R}^{n} \times \mathbb{R}^{n}\right) \backslash(\mathcal{C} \Omega \times \mathcal{C} \Omega), d x d y\right)$,

(here $\mathcal{C} \Omega:=\mathbb{R}^{n} \backslash \Omega$ ). Also, we denote by $X_{0}$ the following linear subspace of $X$

$$
X_{0}=\left\{g \in X: g=0 \text { a.e. in } \mathbb{R}^{n} \backslash \Omega\right\} .
$$

We remark that $X$ and $X_{0}$ are non-empty, since $C_{0}^{2}(\Omega) \subseteq X_{0}$ by [15, Lemma 11].

With these two definitions and condition (1.5) we can write the weak formulation of (1.1), given by the following problem

$$
\left\{\begin{aligned}
\int_{\mathbb{R}^{n} \times \mathbb{R}^{n}} & (u(x)-u(y))(\varphi(x)-\varphi(y)) K(x-y) d x d y+\int_{\Omega} q(x) u(x) \varphi(x) d x \\
& =\lambda \int_{\Omega} u(x) \varphi(x) d x+\int_{\Omega} f(u(x)) \varphi(x) d x+\int_{\Omega} h(x) \varphi(x) d x \quad \forall \varphi \in X_{0} \\
u \in X_{0} . &
\end{aligned}\right.
$$

Before stating our existence result, we would like to note that, in general, the trivial function $u \equiv 0$ is not a solution of problem (1.1). On the other hand, if $h \equiv 0$ and $f(0)=0$, then $u \equiv 0$ solves the problem.

Now, we can state our main result as follows:

Theorem 1. Let $s \in(0,1), n>2 s$ and $\Omega$ be an open, bounded subset of $\mathbb{R}^{n}$ with Lipschitz boundary. Let $K: \mathbb{R}^{n} \backslash\{0\} \rightarrow(0,+\infty)$ be a function satisfying (1.3)-(1.5) and let $f, q$ and $h$ be three functions verifying (1.8)-(1.11).

Then, problem (1.1) admits a solution $u \in X_{0}$ provided either

- $\lambda$ is not an eigenvalue of problem (1.12), or

- $\lambda$ is an eigenvalue of problem (1.12) satisfying (1.13) and conditions (1.14) and (1.15) hold true.

The proof of Theorem 1 is based on variational techniques. Precisely, we will find solutions of problem (1.1) as critical points of the Euler-Lagrange functional naturally associated with the problem. To this purpose we will perform the Saddle Point Theorem by Rabinowitz, see [12, Theorem 4.6]. Hence, as usual, we have to study both the compactness properties of the functional associated with the problem and also its geometrical structure. In doing this we need to consider separately the case when the parameter $\lambda$ is an eigenvalue of $-\mathcal{L}_{K}+q$ and the case when it does not, namely the resonant and the non-resonant situation.

The resonant setting is more difficult to be treated than the non-resonant one. As we said before, in order to manage this case, along the paper we will need an additional property on the parameter $\lambda$, related to the nodal set of the eigenfunctions associated with $\lambda$ (see $(1.13))$.

In the standard case of the Laplacian, this property represents the key-point in the proof of the existence result (see, for instance, [1, Theorem 4.4.11 and Theorem 4.4.17]). Due to the generality of our non-local framework, it is an open problem whether or not all the eigenfunctions of $-\mathcal{L}_{K}+q$ satisfy this property. In the case of the Laplacian all the eigenfunctions verify this condition on the nodal set and this is a direct consequence of a 
Unique Continuation Principle (see, for instance, $[6,10]$ ). As far as we know, there is not a non-local counterpart of this continuation property.

Finally, we would like to point out that the resonant assumption affects both the compactness property and the geometry of the functional. For this reason, the extra assumptions (1.13)-(1.15) (in particular (1.13) and the Landesman-Lazer condition) will be crucial both in proving the compactness and in showing the geometric properties possessed by the Euler-Lagrange functional associated with problem (1.1).

Theorem 1 extends the result obtained in [1, Theorem 4.4.11 and Theorem 4.4.17] (see also [1, Chapter 4] and references therein) in the case of the classical Laplacian operator to a general non-local framework.

The paper is organized as follows. In Section 2 we will give some definitions related to the functional setting we will work in. In Section 3 we will discuss the variational formulation of the problem, while Sections 4 and 5 will be devoted to the proof of Theorem 1, respectively in the non-resonant case and in the resonant one. Finally, in the Appendix A we will briefly discuss the eigenvalue problem (1.12).

\section{The FunCtional AnAlytic Setting}

Here we recall some preliminary results on the functional spaces $X$ and $X_{0}$, whose definitions were recalled in the Introduction. The readers familiar with this topic may skip it and go directly to Section 3 . In the sequel we denote by $Q=\left(\mathbb{R}^{n} \times \mathbb{R}^{n}\right) \backslash \mathcal{O}$, where

$$
\mathcal{O}=(\mathcal{C} \Omega) \times(\mathcal{C} \Omega) \subset \mathbb{R}^{n} \times \mathbb{R}^{n} \text { and } \mathcal{C} \Omega=\mathbb{R}^{n} \backslash \Omega .
$$

The space $X$ is endowed with the norm defined as

$$
\|g\|_{X}=\|g\|_{L^{2}(\Omega)}+\left(\int_{Q}|g(x)-g(y)|^{2} K(x-y) d x d y\right)^{1 / 2},
$$

while we equip $X_{0}$ with the following norm

$$
\|g\|_{X_{0}, q}=\left(\int_{Q}|g(x)-g(y)|^{2} K(x-y) d x d y+\int_{\Omega} q(x)|g(x)|^{2} d x\right)^{1 / 2},
$$

which is equivalent to the usual one defined in (2.1), as we prove in the following lemma:

Lemma 2. Let $K: \mathbb{R}^{n} \backslash\{0\} \rightarrow(0,+\infty)$ be a function satisfying assumptions (1.3)-(1.5) and let $q$ satisfy (1.10). Then, the expression

$$
\langle u, v\rangle_{X_{0}, q}=\int_{Q}(u(x)-u(y))(v(x)-v(y)) K(x-y) d x d y+\int_{\Omega} q(x) u(x) v(x) d x
$$

defines on $X_{0}$ a scalar product that induces a norm, denoted with $\|\cdot\|_{X_{0}, q}$, equivalent to the usual one defined in (2.1).

Proof. Since the expression (2.3) is a sum of two scalar products, it is immediate to observe that $\langle\cdot, \cdot\rangle_{X_{0}, q}$ is a scalar product on $X_{0}$ which induces the norm defined in (2.2).

Now, we show that the norm defined in (2.2) is equivalent to the one given in (2.1). For this, let $v \in X_{0}$. It is easily seen that

$$
\begin{aligned}
& \|v\|_{X_{0}, q}^{2}=\int_{Q}|v(x)-v(y)|^{2} K(x-y) d x d y+\int_{\Omega} q(x)|v(x)|^{2} d x \\
& \leqslant \int_{Q}|v(x)-v(y)|^{2} K(x-y) d x d y+\|q\|_{L^{\infty}(\Omega)}\|v\|_{L^{2}(\Omega)}^{2} \leqslant C_{1}\|v\|_{X}^{2},
\end{aligned}
$$

where $C_{1}=\max \left\{1,\|q\|_{L^{\infty}(\Omega)}\right\}>0$.

Moreover, by [16, Lemma 6] we know that there is a constant $C_{2}>1$ such that

$$
\|v\|_{X}^{2} \leqslant C_{2} \int_{Q}|v(x)-v(y)|^{2} K(x-y) d x d y,
$$


so that, by using also (1.10), we get

$$
\begin{aligned}
& \frac{1}{C_{2}}\|v\|_{X}^{2} \leqslant \int_{Q}|v(x)-v(y)|^{2} K(x-y) d x d y \\
& \leqslant \int_{Q}|v(x)-v(y)|^{2} K(x-y) d x d y+\int_{\Omega} q(x)|v(x)|^{2} d x=\|v\|_{X_{0}, q}^{2} .
\end{aligned}
$$

By combining (2.4) and (2.5) we conclude the proof.

In the following we denote by $H^{s}(\Omega)$ the usual fractional Sobolev space endowed with the norm (the so-called Gagliardo norm)

$$
\|g\|_{H^{s}(\Omega)}=\|g\|_{L^{2}(\Omega)}+\left(\int_{\Omega \times \Omega} \frac{|g(x)-g(y)|^{2}}{|x-y|^{n+2 s}} d x d y\right)^{1 / 2} .
$$

We remark that, even in the model case in which $K(x)=|x|^{-(n+2 s)}$, the norms in (2.1) and (2.6) are not the same, because $\Omega \times \Omega$ is strictly contained in $Q$. This is the reason why the classical fractional Sobolev space is not enough for studying our problem and why we work in the new spaces $X$ and $X_{0}$.

For further details on the fractional Sobolev spaces we refer to [7] and to the references therein, while for other details on $X$ and $X_{0}$ we refer to [15], where these spaces were introduced, and also to $[13,16,17,18,19,20]$, where various properties of these spaces were proved.

\section{VARIATIONAL FORMULATION OF THE PROBLEM}

For the proof of our main result, stated in Theorem 1, we first observe that problem (1.1) has a variational structure. Indeed, the weak formulation of problem (1.1), given in (1.16), represents the Euler-Lagrange equation of the functional $\mathcal{J}: X_{0} \rightarrow \mathbb{R}$ defined as follows

$$
\begin{array}{r}
\mathcal{J}(u)=\frac{1}{2} \int_{\mathbb{R}^{n} \times \mathbb{R}^{n}}|u(x)-u(y)|^{2} K(x-y) d x d y+\frac{1}{2} \int_{\Omega} q(x)|u(x)|^{2} d x \\
-\frac{\lambda}{2} \int_{\Omega}|u(x)|^{2} d x-\int_{\Omega} F(u(x)) d x-\int_{\Omega} h(x) u(x) d x,
\end{array}
$$

where $F(t)=\int_{0}^{t} f(\tau) d \tau$.

Note that the functional $\mathcal{J}$ is well defined thanks to Lemma 2, the definition of $F$, assumptions (1.9)-(1.11) and since $X_{0} \subseteq L^{2}(\Omega) \subseteq L^{1}(\Omega)$ (being $\Omega$ bounded). Moreover, $\mathcal{J}$ is Fréchet differentiable at $u \in X_{0}$ and for any $\varphi \in X_{0}$

$$
\begin{array}{r}
\left\langle\mathcal{J}^{\prime}(u), \varphi\right\rangle=\int_{\mathbb{R}^{n} \times \mathbb{R}^{n}}(u(x)-u(y))(\varphi(x)-\varphi(y)) K(x-y) d x d y+\int_{\Omega} q(x) u(x) \varphi(x) d x \\
-\lambda \int_{\Omega} u(x) \varphi(x) d x-\int_{\Omega} f(u(x)) \varphi(x) d x-\int_{\Omega} h(x) \varphi(x) d x .
\end{array}
$$

Thus, critical points of $\mathcal{J}$ are weak solutions to problem (1.1), that is solutions of (1.16).

At first, we need some notation. In what follows we will denote by

$$
\lambda_{1}<\lambda_{2} \leqslant \ldots \leqslant \lambda_{k} \leqslant \ldots
$$

the sequence of the eigenvalues of $-\mathcal{L}_{K}+q$ (see problem (1.12)), while $e_{k}$ will be the $k$-th eigenfunction corresponding to the eigenvalue $\lambda_{k}$. Moreover, we will set

$$
\mathbb{P}_{k+1}:=\left\{u \in X_{0}:\left\langle u, e_{j}\right\rangle_{X_{0}, q}=0 \quad \forall j=1, \ldots, k\right\}
$$

as defined in Proposition 14 (see Appendix A), while

$$
H_{k}:=\operatorname{span}\left\{e_{1}, \ldots, e_{k}\right\}
$$


will denote the linear subspace generated by the first $k$ eigenfunctions of $-\mathcal{L}_{K}+q$ for any $k \in \mathbb{N}$.

In order to prove Theorem 1 we need some preliminary lemmas.

Lemma 3. The following inequality holds true

$$
\|u\|_{X_{0}, q}^{2} \leqslant \lambda_{k}\|u\|_{L^{2}(\Omega)}^{2}
$$

for all $u \in H_{k}$ and any $k \in \mathbb{N}$.

Proof. Let $u \in H_{k}$. Then, we can write

$$
u(x)=\sum_{i=1}^{k} u_{i} e_{i}(x)
$$

with $u_{i} \in \mathbb{R}, i=1, \ldots, k$.

Since $\left\{e_{1}, \ldots, e_{k}, \ldots\right\}$ is an orthonormal basis of $L^{2}(\Omega)$ and an orthogonal one of $X_{0}$ (see Proposition 14-vi)), by Proposition 14-iv) and $v$ ), we get

$$
\|u\|_{X_{0}, q}^{2}=\sum_{i=1}^{k} u_{i}^{2}\left\|e_{i}\right\|_{X_{0}, q}^{2}=\sum_{i=1}^{k} \lambda_{i} u_{i}^{2} \leqslant \lambda_{k} \sum_{i=1}^{k} u_{i}^{2}=\lambda_{k}\|u\|_{L^{2}(\Omega)}^{2},
$$

which gives the desired assertion.

Lemma 4. The following inequality holds true

$$
\|u\|_{X_{0}, q}^{2} \geqslant \lambda_{k+1}\|u\|_{L^{2}(\Omega)}^{2}
$$

for all $u \in \mathbb{P}_{k+1}$ and any $k \in \mathbb{N}$.

Proof. If $u \equiv 0$, then the assertion is trivial, while if $u \in \mathbb{P}_{k+1} \backslash\{0\}$ it follows from the variational characterization of $\lambda_{k+1}$ given in Proposition 14-iv).

To conclude this section we prove the following result:

Lemma 5. Let $f$ and $h$ be functions verifying (1.8)-(1.9) and (1.11), respectively. Then, there exists a positive constant $\tilde{C}$ such that

$$
\left|\int_{\Omega} F(u(x)) d x+\int_{\Omega} h(x) u(x) d x\right| \leqslant \tilde{C}\|u\|_{X_{0}, q}
$$

for all $u \in X_{0}$.

Proof. By (1.9), (1.11), the definition of $F$, the Hölder inequality, Lemma 2 and [16, Lemma 6], we get

$$
\begin{aligned}
\left|\int_{\Omega} F(u(x)) d x+\int_{\Omega} h(x) u(x) d x\right| & \leqslant M \int_{\Omega}|u(x)| d x+\|h\|_{L^{2}(\Omega)}\|u\|_{L^{2}(\Omega)} \\
& \leqslant M|\Omega|^{1 / 2}\|u\|_{L^{2}(\Omega)}+\tilde{\kappa}\|h\|_{L^{2}(\Omega)}\|u\|_{X_{0}, q} \\
& \leqslant \tilde{C}\|u\|_{X_{0}, q},
\end{aligned}
$$

for a suitable $\tilde{C}>0$ (here $|\Omega|$ denotes the measure of $\Omega$ and $\tilde{\kappa}$ is a positive constant). This gives the desired assertion.

Due to the variational nature of the problem, in order to find weak solutions for problem (1.1), in the following we will look for critical points of the functional $\mathcal{J}$ defined in (3.1). In doing this we need to study separately the resonant case and the non-resonant one, that is the case when the parameter $\lambda$ is an eigenvalue of the operator $-\mathcal{L}_{K}+q$ and the one where $\lambda$ is different from these eigenvalues, respectively. We will treat the non-resonant case in the forthcoming Section 4 and the resonant one in the next Section 5 . 


\section{The NON-RESONANT CASE}

In this section we will prove Theorem 1 in the case when the parameter $\lambda$ appearing in problem (1.1) is not an eigenvalue of the operator $-\mathcal{L}_{K}+q$. As we said before, the idea is to find critical points of the functional $\mathcal{J}$, given in formula (3.1). To this purpose, we will consider two different cases:

- $\lambda<\lambda_{1}$ : in this setting the existence of a solution for problem (1.1) follows from the Weierstrass Theorem (i.e. by direct minimization);

- $\lambda>\lambda_{1}$ : in this framework we will apply the Saddle Point Theorem (see [12]) to the functional $\mathcal{J}$. As usual, for this we have to check that the functional $\mathcal{J}$ has a particular geometric structure (as stated, e.g., in conditions $\left(I_{3}\right)$ and $\left(I_{4}\right)$ of $[12$, Theorem 4.6]) and that it satisfies the Palais-Smale compactness condition (see, for instance, [12, page 3$])$.

4.1. The case $\lambda<\lambda_{1}$. In this subsection, in order to apply the Weierstrass Theorem, we first verify that the functional $\mathcal{J}$ satisfies some geometric features. For this we need a preliminary lemma.

Lemma 6. Let $\lambda<\lambda_{1}$ and let $K: \mathbb{R}^{n} \backslash\{0\} \rightarrow(0,+\infty)$ satisfy assumptions (1.3)-(1.5). Moreover, let $f, q, h$ be functions satisfying conditions (1.8)-(1.11). Then, the functional $\mathcal{J}$ verifies

$$
\liminf _{\|u\|_{X_{0}, q} \rightarrow+\infty} \frac{\mathcal{J}(u)}{\|u\|_{X_{0}, q}^{2}}>0 .
$$

Proof. By the variational characterization of $\lambda_{1}$ given in Proposition 14-i), we get

$$
\lambda_{1}\|u\|_{L^{2}}^{2} \leqslant\|u\|_{X_{0}, q}^{2}
$$

for any $u \in X_{0}$ (of course, if $u \equiv 0$, this inequality is trivial).

Hence, as a consequence of this and Lemma 5 , we get

$$
\begin{aligned}
\mathcal{J}(u) & =\frac{1}{2}\|u\|_{X_{0}, q}^{2}-\frac{\lambda}{2} \int_{\Omega}|u(x)|^{2} d x-\int_{\Omega} F(u(x)) d x-\int_{\Omega} h(x) u(x) d x \\
& \geqslant \begin{cases}\frac{1}{2}\left(1-\frac{\lambda}{\lambda_{1}}\right)\|u\|_{X_{0}, q}^{2}-\tilde{C}\|u\|_{X_{0}, q} & \text { if } \lambda>0 \\
\frac{1}{2}\|u\|_{X_{0}, q}^{2}-\tilde{C}\|u\|_{X_{0}, q} & \text { if } \lambda \leqslant 0,\end{cases}
\end{aligned}
$$

so that, dividing by $\|u\|_{X_{0}, q}^{2}$ and passing to the limit as $\|u\|_{X_{0}, q} \rightarrow+\infty$, we get the assertion, since $\lambda<\lambda_{1}$ by assumption.

4.2. Proof of Theorem 1 in the non-resonant case, when $\lambda<\lambda_{1}$. Let us note that the map

$$
u \mapsto\|u\|_{X_{0}, q}^{2}
$$

is lower semicontinuous in the weak topology of $X_{0}$, while the map

$$
u \mapsto \int_{\Omega} F(x, u(x)) d x
$$

is continuous in the weak topology of $X_{0}$. Indeed, if $\left\{u_{j}\right\}_{j \in \mathbb{N}}$ is a sequence in $X_{0}$ such that $u_{j} \rightarrow u$ in $X_{0}$, then, by [16, Lemma 8] and [3, Theorem IV.9], up to a subsequence, $u_{j}$ converges to $u$ strongly in $L^{\nu}(\Omega)$ and a.e. in $\Omega$ and it is dominated by some function $\kappa_{\nu} \in L^{\nu}(\Omega)$ for any $\nu \in\left[1,2^{*}\right)$. Here and in the following $2^{*}$ is the fractional critical Sobolev exponent given $b y^{2}$

$$
2^{*}=\frac{2 n}{n-2 s} .
$$

\footnotetext{
${ }^{2}$ Note that, when $s=1$ the exponent $2^{*}$ reduces to the classical critical Sobolev exponent $2 *=2 n /(n-2)$.
} 
Then, by (1.8) and (1.9) it follows

$$
F\left(u_{j}(x)\right) \rightarrow F(u(x)) \text { a.e. } x \in \Omega
$$

as $j \rightarrow+\infty$ and

$$
\left|F\left(u_{j}(x)\right)\right| \leqslant M\left|u_{j}(x)\right| \leqslant M \kappa_{1}(x) \in L^{1}(\Omega)
$$

a.e. $x \in \Omega$ and for any $j \in \mathbb{N}$. Hence, by applying the Lebesgue Dominated Convergence Theorem applied in $L^{1}(\Omega)$, we have that

$$
\int_{\Omega} F\left(u_{j}(x)\right) d x \rightarrow \int_{\Omega} F(u(x)) d x
$$

as $j \rightarrow+\infty$, that is the map

$$
u \mapsto \int_{\Omega} F(x, u(x)) d x
$$

is continuous from $X_{0}$ with the weak topology to $\mathbb{R}$.

Moreover, again by [16, Lemma 8], also the map

$$
u \mapsto \frac{\lambda}{2} \int_{\Omega}|u(x)|^{2} d x+\int_{\Omega} h(x) u(x) d x
$$

is continuous in the weak topology of $X_{0}$. Hence, the functional $\mathcal{J}$ is lower semicontinuous in the weak topology of $X_{0}$.

Furthermore, Lemma 6 gives the coerciveness of $\mathcal{J}$. Thus, we can apply the Weierstrass Theorem in order to find a minimum $u$ of $\mathcal{J}$ on $X_{0}$. Clearly, $u$ is a weak solution of problem (1.1).

4.3. The case $\lambda>\lambda_{1}$. In this subsection we can suppose that $\lambda_{k}<\lambda<\lambda_{k+1}$ for some $k \in \mathbb{N}$. This is due to the fact that the sequence of eigenvalues $\lambda_{k}$ of the operator $-\mathcal{L}_{K}+q$ diverges to $+\infty$ as $k \rightarrow+\infty$ (see Proposition 14-iv)) .

In this framework we will look for critical points of the functional $\mathcal{J}$ using the Saddle Point Theorem. First of all, we need some preliminary lemmas.

Lemma 7. Let $\lambda \in\left(\lambda_{k}, \lambda_{k+1}\right]$ for some $k \in \mathbb{N}$. Let $K: \mathbb{R}^{n} \backslash\{0\} \rightarrow(0,+\infty)$ satisfy assumptions (1.3)-(1.5) and let $f, q$ and $h$ be functions satisfying (1.8)-(1.11). Then, the functional $\mathcal{J}$ verifies

$$
\limsup _{\substack{u \in H_{k} \\\|u\|_{X_{0}, q} \rightarrow+\infty}} \frac{\mathcal{J}(u)}{\|u\|_{X_{0}, q}^{2}}<0 .
$$

Proof. Let $u \in H_{k}$. By Lemma 3, Lemma 5 and the fact that $\lambda>0$ (being $\lambda>\lambda_{k} \geqslant \lambda_{1}>0$ ) we get

$$
\begin{aligned}
\mathcal{J}(u) & =\frac{1}{2}\|u\|_{X_{0}, q}^{2}-\frac{\lambda}{2} \int_{\Omega}|u(x)|^{2} d x-\int_{\Omega} F(u(x)) d x-\int_{\Omega} h(x) u(x) d x \\
& \leqslant \frac{1}{2}\left(1-\frac{\lambda}{\lambda_{k}}\right)\|u\|_{X_{0}, q}^{2}+\tilde{C}\|u\|_{X_{0}, q} .
\end{aligned}
$$

So, dividing by $\|u\|_{X_{0}, q}^{2}$ and passing to the limit as $\|u\|_{X_{0}, q} \rightarrow+\infty$, we get the assertion, since $\lambda>\lambda_{k}$.

Note that Lemma 7 holds true for any $\lambda \in\left(\lambda_{k}, \lambda_{k+1}\right]$ for some $k \in \mathbb{N}$ and this will be used in the resonant case of problem (1.1), that is in the case when $\lambda=\lambda_{k+1}$.

Lemma 8. Let $\lambda \in\left(\lambda_{k}, \lambda_{k+1}\right)$ for some $k \in \mathbb{N}$. Let $K: \mathbb{R}^{n} \backslash\{0\} \rightarrow(0,+\infty)$ satisfy assumptions (1.3)-(1.5) and let $f, q$ and $h$ be functions satisfying (1.8)-(1.11). Then, the functional $\mathcal{J}$ verifies

$$
\liminf _{\substack{u \in \mathbb{P}_{k+1} \\\|u\|_{X_{0}, q} \rightarrow+\infty}} \frac{\mathcal{J}(u)}{\|u\|_{X_{0}, q}^{2}}>0 .
$$


Proof. Let $u \in \mathbb{P}_{k+1}$. In this case, by Lemma 4, Lemma 5 and the positivity of $\lambda$, we have

$$
\mathcal{J}(u) \geqslant \frac{1}{2}\left(1-\frac{\lambda}{\lambda_{k+1}}\right)\|u\|_{X_{0}, q}^{2}-\tilde{C}\|u\|_{X_{0}, q}
$$

so that, dividing by $\|u\|_{X_{0}, q}^{2}$ and passing to the limit as $\|u\|_{X_{0}, q} \rightarrow+\infty$, we get the assertion, being $\lambda<\lambda_{k+1}$.

With these preliminary results we can prove that the functional $\mathcal{J}$ has the geometric structure required by the Saddle Point Theorem, according to the following result:

Proposition 9. Let $\lambda \in\left(\lambda_{k}, \lambda_{k+1}\right)$ for some $k \in \mathbb{N}$. Let $K: \mathbb{R}^{n} \backslash\{0\} \rightarrow(0,+\infty)$ satisfy assumptions (1.3)-(1.5) and let $f, q$ and $h$ be functions satisfying (1.8)-(1.11). Then, there exist two positive constants $C$ and $T$ such that

$$
\sup _{\substack{u \in H_{k} \\\|u\|_{X_{0}, q}=T}} \mathcal{J}(u)<-C \leqslant \inf _{u \in \mathbb{P}_{k+1}} \mathcal{J}(u) .
$$

Proof. By Lemma 8 it follows that for any $H>0$ there exists $R>0$ such that if $u \in \mathbb{P}_{k+1}$ and $\|u\|_{X_{0}} \geqslant R$ then $\mathcal{J}(u) \geqslant H$.

On the other hand, if $u \in \mathbb{P}_{k+1}$ with $\|u\|_{X_{0}, q}<R$, by applying Lemma 5 , the Hölder inequality, Lemma 2 and [16, Lemma 6] we have

$$
\begin{aligned}
\mathcal{J}(u) & \geqslant-\frac{\lambda}{2} \int_{\Omega}|u(x)|^{2} d x-\int_{\Omega} F(u(x)) d x-\int_{\Omega} h(x) u(x) d x \geqslant \\
& \geqslant-\bar{\kappa}\|u\|_{X_{0}, q}^{2}-\tilde{C}\|u\|_{X_{0}, q} \\
& >-\bar{\kappa} R^{2}-\tilde{C} R=:-C,
\end{aligned}
$$

thanks to the fact that $\lambda>0$ (being $\lambda>\lambda_{k} \geqslant \lambda_{1}>0$ by Proposition 14-i)). Also, here $\bar{\kappa}$ is a positive constant.

So, we get

$$
\mathcal{J}(u) \geqslant-C \quad \text { for any } u \in \mathbb{P}_{k+1} .
$$

Moreover, by Lemma 7 there exists $T>0$ such that for any $u \in H_{k}$ with $\|u\|_{X_{0}, q} \geqslant T$ we have

$$
\sup _{\substack{u \in H_{k} \\\|u\|_{X_{0}, q}=T}} \mathcal{J}(u) \leqslant \sup _{\substack{u \in H_{k} \\\|u\|_{X_{0}, q} \geqslant T}} \mathcal{J}(u)<-C .
$$

Thus, Proposition 9 follows from (4.2) and (4.3).

Roughly speaking, Proposition 9 says that $\mathcal{J}$ has the geometric structure required by the Saddle Point Theorem.

Finally, we have to show that $\mathcal{J}$ satisfies the Palais-Smale condition. To this purpose, first of all we prove that every Palais-Smale sequence for $\mathcal{J}$ is bounded in $X_{0}$.

Proposition 10. Let $\lambda \in\left(\lambda_{k}, \lambda_{k+1}\right)$ for some $k \in \mathbb{N}$. Let $K: \mathbb{R}^{n} \backslash\{0\} \rightarrow(0,+\infty)$ satisfy assumptions (1.3)-(1.5) and let $f, q$ and $h$ be functions satisfying $(1.8)-(1.11)$. Let $c \in \mathbb{R}$ and let $\left\{u_{j}\right\}_{j \in \mathbb{N}}$ be a sequence in $X_{0}$ such that

$$
\mathcal{J}\left(u_{j}\right) \leqslant c
$$

and

$$
\sup \left\{\left|\left\langle\mathcal{J}^{\prime}\left(u_{j}\right), \varphi\right\rangle\right|: \varphi \in X_{0},\|\varphi\|_{X_{0}, q}=1\right\} \rightarrow 0
$$

as $j \rightarrow+\infty$. Then, the sequence $\left\{u_{j}\right\}_{j \in \mathbb{N}}$ is bounded in $X_{0}$. 
Proof. We argue by contradiction and we suppose that the sequence $\left\{u_{j}\right\}_{j \in \mathbb{N}}$ is unbounded in $X_{0}$. As a consequence, up to a subsequence, we can assume that

$$
\left\|u_{j}\right\|_{X_{0}, q} \rightarrow+\infty \text { as } j \rightarrow+\infty \text {. }
$$

Thus, there exists $u \in X_{0}$ such that $u_{j} /\left\|u_{j}\right\|_{X_{0}, q}$ converges to $u$ weakly in $X_{0}$, that is

$$
\begin{aligned}
& \int_{\mathbb{R}^{n} \times \mathbb{R}^{n}}\left(\frac{u_{j}(x)}{\left\|u_{j}\right\|_{X_{0}, q}}-\frac{u_{j}(y)}{\left\|u_{j}\right\|_{X_{0}, q}}\right)(\varphi(x)-\varphi(y)) K(x-y) d x d y+\int_{\Omega} q(x) \frac{u_{j}(x)}{\left\|u_{j}\right\|_{X_{0}, q}} \varphi(x) d x \\
& \rightarrow \int_{\mathbb{R}^{n} \times \mathbb{R}^{n}}(u(x)-u(y))(\varphi(x)-\varphi(y)) K(x-y) d x d y+\int_{\Omega} q(x) u(x) \varphi(x) d x
\end{aligned}
$$

as $j \rightarrow+\infty$, for any $\varphi \in X_{0}$.

Hence, by applying [16, Lemma 8] and [3, Theorem IV.9], up to a subsequence

$$
\begin{aligned}
& \frac{u_{j}}{\left\|u_{j}\right\|_{X_{0}, q}} \rightarrow u \text { in } L^{\nu}\left(\mathbb{R}^{n}\right) \text { for any } \nu \in\left[1,2^{*}\right) \\
& \frac{u_{j}}{\left\|u_{j}\right\|_{X_{0}, q}} \rightarrow u \quad \text { a.e. in } \mathbb{R}^{n}
\end{aligned}
$$

as $j \rightarrow+\infty$. Here $2^{*}$ is the exponent defined as in (4.1).

Furthermore, by (1.9), (1.11) and the Hölder inequality it follows that

$$
\begin{aligned}
& \frac{1}{\left\|u_{j}\right\|_{X_{0}, q}}\left|\int_{\Omega} f\left(u_{j}(x)\right) \varphi(x) d x+\int_{\Omega} h(x) \varphi(x) d x\right| \\
& \leqslant \frac{1}{\left\|u_{j}\right\|_{X_{0}, q}}\left(M\|\varphi\|_{L^{1}(\Omega)}+\|h\|_{L^{2}(\Omega)}\|\varphi\|_{L^{2}(\Omega)}\right) \rightarrow 0
\end{aligned}
$$

as $j \rightarrow+\infty$, for any $\varphi \in X_{0}$, thanks to (4.6).

So, by (4.7)-(4.9) we have

$$
\begin{gathered}
\frac{\left\langle\mathcal{J}^{\prime}\left(u_{j}\right), \varphi\right\rangle}{\left\|u_{j}\right\|_{X_{0}, q}} \rightarrow \int_{\mathbb{R}^{n} \times \mathbb{R}^{n}}(u(x)-u(y))(\varphi(x)-\varphi(y)) K(x-y) d x d y \\
+\int_{\Omega} q(x) u(x) \varphi(x) d x-\lambda \int_{\Omega} u(x) \varphi(x) d x
\end{gathered}
$$

as $j \rightarrow+\infty$, for any $\varphi \in X_{0}$.

Hence, by combining (4.5), (4.6) and (4.10) we get

$\int_{\mathbb{R}^{n} \times \mathbb{R}^{n}}(u(x)-u(y))(\varphi(x)-\varphi(y)) K(x-y) d x d y+\int_{\Omega} q(x) u(x) \varphi(x) d x=\lambda \int_{\Omega} u(x) \varphi(x) d x$

for all $\varphi \in X_{0}$ and we deduce that $u$ is a weak solution of problem (1.12).

Let us now prove that $u \not \equiv 0$ in $X_{0}$. Assume, by contradiction, that $u \equiv 0$ in $X_{0}$. By (4.5) with $\varphi=u_{j} /\left\|u_{j}\right\|_{X_{0}, q}$ we get

$$
\begin{gathered}
\int_{\mathbb{R}^{n} \times \mathbb{R}^{n}} \frac{\left|u_{j}(x)-u_{j}(y)\right|^{2}}{\left\|u_{j}\right\|_{X_{0}, q}} K(x-y) d x d y+\int_{\Omega} q(x) \frac{\left|u_{j}(x)\right|^{2}}{\left\|u_{j}\right\|_{X_{0}, q}} d x-\lambda \int_{\Omega} \frac{\left|u_{j}(x)\right|^{2}}{\left\|u_{j}\right\|_{X_{0}, q}} d x \\
-\int_{\Omega} f\left(u_{j}(x)\right) \frac{u_{j}(x)}{\left\|u_{j}\right\|_{X_{0}, q}} d x-\int_{\Omega} h(x) \frac{u_{j}(x)}{\left\|u_{j}\right\|_{X_{0}, q}} d x \rightarrow 0
\end{gathered}
$$

as $j \rightarrow+\infty$. Moreover, by (1.9), (1.11) and (4.8), since $u \equiv 0$, we get

$$
\begin{aligned}
& \left|\int_{\Omega} f\left(u_{j}(x)\right) \frac{u_{j}(x)}{\left\|u_{j}\right\|_{X_{0}, q}} d x+\int_{\Omega} h(x) \frac{u_{j}(x)}{\left\|u_{j}\right\|_{X_{0}, q}} d x\right| \\
& \leqslant M \frac{\left\|u_{j}\right\|_{L^{1}(\Omega)}}{\left\|u_{j}\right\|_{X_{0}, q}}+\frac{\|h\|_{L^{2}(\Omega)}\left\|u_{j}\right\|_{L^{2}(\Omega)}}{\left\|u_{j}\right\|_{X_{0}, q}} \rightarrow 0
\end{aligned}
$$


as $j \rightarrow+\infty$.

Hence, by combining (4.11) and (4.12) it follows that

$$
\int_{\mathbb{R}^{n} \times \mathbb{R}^{n}} \frac{\left|u_{j}(x)-u_{j}(y)\right|^{2}}{\left\|u_{j}\right\|_{X_{0}, q}} K(x-y) d x d y+\int_{\Omega} q(x) \frac{\left|u_{j}(x)\right|^{2}}{\left\|u_{j}\right\|_{X_{0}, q}} d x-\lambda \int_{\Omega} \frac{\left|u_{j}(x)\right|^{2}}{\left\|u_{j}\right\|_{X_{0}, q}} d x \rightarrow 0
$$

so that, dividing by $\left\|u_{j}\right\|_{X_{0}, q}$, we get

$$
1-\lambda \frac{\left\|u_{j}\right\|_{L^{2}(\Omega)}^{2}}{\left\|u_{j}\right\|_{X_{0}, q}^{2}} \rightarrow 0 \quad \text { as } \quad j \rightarrow+\infty .
$$

This gives $1=0$, again by (4.8) and the fact that $u \equiv 0$ in $X_{0}$. Of course, this is a contradiction and so $u \not \equiv 0$ in $X_{0}$.

In this way we have constructed a non-trivial function $u$ solving (1.12), but this contradicts the non-resonance assumption $\lambda_{k}<\lambda<\lambda_{k+1}$. Thus, the sequence $\left\{u_{j}\right\}_{j \in \mathbb{N}}$ is bounded in $X_{0}$ and this ends the proof of Proposition 10 .

Now, we can prove the following result, whose proof is quite standard and, differently from Proposition 10, it is not affected by the resonant/non-resonant assumptions:

Proposition 11. Let $\lambda \in \mathbb{R}$. Let $K: \mathbb{R}^{n} \backslash\{0\} \rightarrow(0,+\infty)$ satisfy assumptions (1.3)-(1.5) and let $f, q$ and $h$ be functions satisfying (1.8)-(1.11). Let $\left\{u_{j}\right\}_{j \in \mathbb{N}}$ be a bounded sequence in $X_{0}$ such that (4.5) holds true. Then, there exists $u_{\infty} \in X_{0}$ such that, up to a subsequence,

$$
\left\|u_{j}-u_{\infty}\right\|_{X_{0}, q} \rightarrow 0 \quad \text { as } j \rightarrow+\infty \text {. }
$$

Proof. Since $\left\{u_{j}\right\}_{j \in \mathbb{N}}$ is bounded by assumption and $X_{0}$ is a reflexive space (being a Hilbert space, by [16, Lemma 7]), up to a subsequence, there exists $u_{\infty} \in X_{0}$ such that $u_{j}$ converges to $u_{\infty}$ weakly in $X_{0}$, that is

$$
\begin{aligned}
& \int_{\mathbb{R}^{n} \times \mathbb{R}^{n}}\left(u_{j}(x)-u_{j}(y)\right)(\varphi(x)-\varphi(y)) K(x-y) d x d y+\int_{\Omega} q(x) u_{j}(x) \varphi(x) d x \rightarrow \\
& \int_{\mathbb{R}^{n} \times \mathbb{R}^{n}}\left(u_{\infty}(x)-u_{\infty}(y)\right)(\varphi(x)-\varphi(y)) K(x-y) d x d y+\int_{\Omega} q(x) u_{\infty}(x) \varphi(x) d x
\end{aligned}
$$

as $j \rightarrow+\infty$, for any $\varphi \in X_{0}$. Moreover, by applying [16, Lemma 8] and [3, Theorem IV.9], up to a subsequence

$$
\begin{array}{ll}
u_{j} \rightarrow u_{\infty} & \text { in } L^{\nu}\left(\mathbb{R}^{n}\right) \text { for any } \nu \in\left[1,2^{*}\right) \\
u_{j} \rightarrow u_{\infty} & \text { a.e. in } \mathbb{R}^{n}
\end{array}
$$

as $j \rightarrow+\infty$. Again $2^{*}$ is defined as in (4.1).

By (4.5) we have

$$
\begin{aligned}
& 0 \leftarrow\left\langle\mathcal{J}^{\prime}\left(u_{j}\right), u_{j}-u_{\infty}\right\rangle=\int_{\mathbb{R}^{n} \times \mathbb{R}^{n}}\left|u_{j}(x)-u_{j}(y)\right|^{2} K(x-y) d x d y \\
& +\int_{\Omega} q(x)\left|u_{j}(x)\right|^{2} d x-\int_{\mathbb{R}^{n} \times \mathbb{R}^{n}}\left(u_{j}(x)-u_{j}(y)\right)\left(u_{\infty}(x)-u_{\infty}(y)\right) K(x-y) d x d y \\
& -\int_{\Omega} q(x) u_{j}(x) u_{\infty}(x) d x-\lambda \int_{\Omega} u_{j}(x)\left(u_{j}(x)-u_{\infty}(x)\right) d x \\
& -\int_{\Omega} f\left(x, u_{j}(x)\right)\left(u_{j}(x)-u_{\infty}(x)\right) d x-\int_{\Omega} h(x)\left(u_{j}(x)-u_{\infty}(x)\right) d x
\end{aligned}
$$

as $j \rightarrow+\infty$.

Also note that, by the definition of norm in $X_{0}$ (see formula $(2.2)$ ), since $\left\{u_{j}\right\}_{j \in \mathbb{N}}$ is bounded in $X_{0}$, then $\left\{u_{j}\right\}_{j \in \mathbb{N}}$ does in $L^{2}(\Omega)$. Hence, by using the Hölder inequality, (1.9), 
(1.11) and (4.14), we get

$$
\begin{aligned}
& \mid \lambda \int_{\Omega} u_{j}(x)\left(u_{j}(x)-u_{\infty}(x)\right) d x+\int_{\Omega} f\left(x, u_{j}(x)\right)\left(u_{j}(x)-u_{\infty}(x)\right) d x \\
&+\int_{\Omega} h(x)\left(u_{j}(x)-u_{\infty}(x)\right) d x \mid \\
& \leqslant\left(\lambda\left\|u_{j}\right\|_{L^{2}(\Omega)}+M|\Omega|^{1 / 2}+\|h\|_{L^{2}(\Omega)}\right)\left\|u_{j}-u_{\infty}\right\|_{L^{2}(\Omega)} \rightarrow 0
\end{aligned}
$$

as $j \rightarrow+\infty$.

Then, by (4.13), (4.15) and (4.16) we obtain

$$
\begin{aligned}
\int_{\mathbb{R}^{n} \times \mathbb{R}^{n}}\left|u_{j}(x)-u_{j}(y)\right|^{2} K(x-y) d x d y+\int_{\Omega} q(x)\left|u_{j}(x)\right|^{2} d x \\
\quad \rightarrow \int_{\mathbb{R}^{n} \times \mathbb{R}^{n}}\left|u_{\infty}(x)-u_{\infty}(y)\right|^{2} K(x-y) d x d y+\int_{\Omega} q(x)\left|u_{\infty}(x)\right|^{2} d x,
\end{aligned}
$$

that is

$$
\left\|u_{j}\right\|_{X_{0}, q} \rightarrow\left\|u_{\infty}\right\|_{X_{0}, q}
$$

as $j \rightarrow+\infty$.

Finally, we have that

$$
\begin{aligned}
& \left\|u_{j}-u_{\infty}\right\|_{X_{0}, q}^{2}=\left\|u_{j}\right\|_{X_{0}, q}^{2}+\left\|u_{\infty}\right\|_{X_{0}, q}^{2} \\
& -2 \int_{\mathbb{R}^{n} \times \mathbb{R}^{n}}\left(u_{j}(x)-u_{j}(y)\right)\left(u_{\infty}(x)-u_{\infty}(y)\right) K(x-y) d x d y-2 \int_{\Omega} q(x) u_{j}(x) u_{\infty}(x) d x \\
& \rightarrow 2\left\|u_{\infty}\right\|_{X_{0}, q}^{2}-2 \int_{\mathbb{R}^{n} \times \mathbb{R}^{n}}\left|u_{\infty}(x)-u_{\infty}(y)\right|^{2} K(x-y) d x d y-2 \int_{\Omega} q(x)\left|u_{\infty}(x)\right|^{2} d x=0
\end{aligned}
$$

as $j \rightarrow+\infty$, again thanks to (4.13) and (4.17). This concludes the proof.

4.4. Proof of Theorem 1 in the non-resonant case, when $\lambda>\lambda_{1}$. For the proof it is enough to observe that, by Proposition 9 the functional $\mathcal{J}$ satisfies the geometric assumptions required by the Saddle Point Theorem, while by Propositions 10 and 11 it verifies the Palais-Smale compactness condition. Hence, as a consequence of the Saddle Point Theorem, $\mathcal{J}$ possesses a critical point $u \in X_{0}$, which, of course, is a weak solution of problem (1.1).

\section{THE RESONANT CASE}

In this section we study problem (1.1) in presence of a resonance, namely when $\lambda$ is an eigenvalue of the operator $-\mathcal{L}_{K}+q$. This kind of problem is harder to solve than the non-resonant one and we have to impose further conditions on the nonlinearities and on the parameter appearing in the equation. Namely, we have to assume the extra conditions (1.14) and (1.15) on $f$ and $h$. Also, we need an additional condition on the parameter $\lambda$, that is $\lambda$ has to verify (1.13). With this respect, we would like to note that assumption (1.13) is satisfied in the model case $-\mathcal{L}_{K}=(-\Delta)^{s}$, when $\lambda$ is the first eigenvalue of this operator, since its first eigenfunction is strictly positive (see [17, Proposition 9 and Appendix A] and [21]).

We also would like to remark that condition (1.13) is compatible with the standard case of the Laplacian, since, in this setting, it is always satisfied. In fact, in this standard situation it is well know that all the eigenfunctions are almost everywhere different from zero. This is a consequence of a Unique Continuation Principle (see $[6,10]$ ), which, as far as we know, it is not available in the non-local framework. We think it could be interesting to investigate this problem.

Without loss of generality, in the sequel we assume that for some $k, m \in \mathbb{N}$

$$
\lambda_{k}<\lambda=\lambda_{k+1}=\ldots=\lambda_{k+m}<\lambda_{k+m+1},
$$


that is we suppose that $\lambda$ is an eigenvalue of $-\mathcal{L}_{K}+q$ with multiplicity $m$.

As in the non-resonant framework, here the idea is to apply the Saddle Point Theorem. Hence, also in this case, we have to check that the functional $\mathcal{J}$ satisfies the Palais-Smale condition and possesses a suitable geometric structure. The resonant assumption (5.1) affects both these problems (i.e. the compactness and the geometric structure of the functional), making the proof more difficult than in the non-resonant setting.

Let us start by proving the compactness condition. If compared with the non-resonant case, in the resonant one the difference lies in the proof of the boundedness of the PalaisSmale sequence. Indeed, in order to show that the Palais-Smale sequence is bounded in $X_{0}$, here we have to use different arguments, since the ones used in the non-resonant case are based mainly on the fact that the parameter $\lambda$ is not an eigenvalue of the operator $-\mathcal{L}_{K}+q$. Precisely, we will argue by contradiction and we will use the Landesman-Lazer condition (1.15), which will be fundamental for our arguments. Also, it will be crucial for our proof the property stated in (1.13).

Proposition 12. Let $\lambda$ be as in (5.1) for some $k, m \in \mathbb{N}$, and verify (1.13). Let $K$ : $\mathbb{R}^{n} \backslash\{0\} \rightarrow(0,+\infty)$ satisfy assumptions (1.3)-(1.5). Moreover, let $f, q$ and $h$ be functions satisfying (1.8)-(1.11), (1.14) and (1.15). Let $c \in \mathbb{R}$ and let $\left\{u_{j}\right\}_{j \in \mathbb{N}}$ be a sequence in $X_{0}$ such that (4.4) and (4.5) hold true. Then, the sequence $\left\{u_{j}\right\}_{j \in \mathbb{N}}$ is bounded in $X_{0}$.

Proof. First of all, let us write $u_{j}=w_{j}+v_{j}$, with $w_{j} \in E_{\lambda}$ and $v_{j} \in E_{\lambda}^{\perp}$, where

$$
E_{\lambda}:=\operatorname{span}\left\{e_{k+1}, \ldots, e_{k+m}\right\}
$$

is the linear space generated by the eigenfunctions related to $\lambda=\lambda_{k+1}$ (see assumption (5.1)).

In order to prove Proposition 12, it is enough to show that both the sequences $\left\{w_{j}\right\}_{j \in \mathbb{N}}$ and $\left\{v_{j}\right\}_{j \in \mathbb{N}}$ are bounded in $X_{0}$.

Let us prove first that the sequence $\left\{v_{j}\right\}_{j \in \mathbb{N}}$ is bounded in $X_{0}$. For this, note that, since $w_{j} \in E_{\lambda}$, then

$$
-\mathcal{L}_{K} w_{j}+q(x) w_{j}=\lambda w_{j}
$$

in the weak sense, that is for any $\varphi \in X_{0}$

$$
\begin{aligned}
\int_{\mathbb{R}^{n} \times \mathbb{R}^{n}}\left(w_{j}(x)-w_{j}(y)\right)(\varphi(x)-\varphi(y)) d x d y & +\int_{\Omega} q(x) w_{j}(x) \varphi(x) d x \\
& -\lambda \int_{\Omega} w_{j}(x) \varphi(x) d x=0 .
\end{aligned}
$$

Moreover, by linearity, for any $\varphi \in X_{0}$

$$
\begin{array}{r}
\int_{\mathbb{R}^{n} \times \mathbb{R}^{n}}\left(u_{j}(x)-u_{j}(y)\right)(\varphi(x)-\varphi(y)) d x d y=\int_{\mathbb{R}^{n} \times \mathbb{R}^{n}}\left(w_{j}(x)-w_{j}(y)\right)(\varphi(x)-\varphi(y)) d x d y \\
+\int_{\mathbb{R}^{n} \times \mathbb{R}^{n}}\left(v_{j}(x)-v_{j}(y)\right)(\varphi(x)-\varphi(y)) d x d y
\end{array}
$$

and

$$
\int_{\Omega} q(x) u_{j}(x) \varphi(x) d x=\int_{\Omega} q(x) w_{j}(x) \varphi(x) d x+\int_{\Omega} q(x) v_{j}(x) \varphi(x) d x .
$$


Hence, as a consequence of (5.2)-(5.4) and (4.5) we get that for any $\varphi \in X_{0}$

$$
\begin{aligned}
& 0 \leftarrow\left\langle\mathcal{J}^{\prime}\left(u_{j}\right), \varphi\right\rangle= \\
& \int_{\mathbb{R}^{n} \times \mathbb{R}^{n}}\left(w_{j}(x)-w_{j}(y)\right)(\varphi(x)-\varphi(y)) K(x-y) d x d y+\int_{\Omega} q(x) w_{j}(x) \varphi(x) d x \\
& \quad+\int_{\mathbb{R}^{n} \times \mathbb{R}^{n}}\left(v_{j}(x)-v_{j}(y)\right)(\varphi(x)-\varphi(y)) K(x-y) d x d y+\int_{\Omega} q(x) v_{j}(x) \varphi(x) d x \\
& \quad-\lambda \int_{\Omega} u_{j}(x) \varphi(x) d x-\int_{\Omega} f\left(u_{j}(x)\right) \varphi(x) d x-\int_{\Omega} h(x) \varphi(x) d x \\
& =\int_{\mathbb{R}^{n} \times \mathbb{R}^{n}}\left(v_{j}(x)-v_{j}(y)\right)(\varphi(x)-\varphi(y)) K(x-y) d x d y+\int_{\Omega} q(x) v_{j}(x) \varphi(x) d x \\
& \quad-\lambda \int_{\Omega} v_{j}(x) \varphi(x) d x-\int_{\Omega} f\left(u_{j}(x)\right) \varphi(x) d x-\int_{\Omega} h(x) \varphi(x) d x
\end{aligned}
$$

as $j \rightarrow+\infty$.

Now, assume by contradiction that $\left\|v_{j}\right\|_{X_{0}, q} \rightarrow+\infty$ as $j \rightarrow+\infty$. Arguing exactly as in the proof of Proposition 10 one shows that $v_{j} /\left\|v_{j}\right\|_{X_{0}, q}$ converges weakly in $X_{0}$ to an eigenfunction $v$ relative to $\lambda$.

Of course $v \in E_{\lambda} \backslash\{0\}$, being an eigenfunction. On the other hand, since

$$
v_{j} \in E_{\lambda}^{\perp}=\overline{\operatorname{span}\left\{e_{1}, \ldots, e_{k}, e_{k+m+1}, \ldots\right\}},
$$

then $v \in E_{\lambda}^{\perp}$. This leads to a contradiction since $v \not \equiv 0$ and $v \in E_{\lambda} \cap E_{\lambda}^{\perp}=\{0\}$. Then, $\left\{v_{j}\right\}_{j \in \mathbb{N}}$ is bounded in $X_{0}$.

Now, it remains to prove that $\left\{w_{j}\right\}_{j \in \mathbb{N}}$ is bounded in $X_{0}$. Also in this case we argue by contradiction and assume that

$$
\left\|w_{j}\right\|_{X_{0}, q} \rightarrow+\infty
$$

as $j \rightarrow+\infty$.

Since $E_{\lambda}$ is finite dimensional, there exists $w \in E_{\lambda}$ such that, up to a subsequence, $w_{j} /\left\|w_{j}\right\|_{X_{0}, q}$ converges to $w$ strongly in $X_{0}$ as $j \rightarrow+\infty$. Moreover, by applying [16, Lemma 8] and [3, Theorem IV.9], up to a subsequence

$$
\begin{aligned}
& \frac{w_{j}}{\left\|w_{j}\right\|_{X_{0}, q}} \rightarrow w \quad \text { in } L^{\nu}\left(\mathbb{R}^{n}\right) \text { for any } \nu \in\left[1,2^{*}\right) \\
& \frac{w_{j}}{\left\|w_{j}\right\|_{X_{0}, q}} \rightarrow w \quad \text { a.e. in } \mathbb{R}^{n}
\end{aligned}
$$

as $j \rightarrow+\infty$. The exponent $2^{*}$ is given in (4.1) .

Note also that, since $w \in E_{\lambda}$, for any $\varphi \in X_{0}$ we get

$$
\begin{aligned}
\int_{\mathbb{R}^{n} \times \mathbb{R}^{n}}(w(x)-w(y))(\varphi(x)-\varphi(y)) K(x-y) d x d y & +\int_{\Omega} q(x) w(x) \varphi(x) d x \\
& =\lambda \int_{\Omega} w(x) \varphi(x) d x
\end{aligned}
$$

that is $w$ is an eigenfunction of problem (1.12). Hence, by (1.13), the function $w$ is almost everywhere different from zero, say

$$
w(x) \neq 0 \text { for any } x \in \Omega \backslash \mathcal{N},
$$

where $\mathcal{N} \subset \Omega$ has zero Lebesgue measure. 
So, by using (5.6), (5.7), the fact that $\left\{v_{j}\right\}_{j \in \mathbb{N}}$ is bounded ${ }^{3}$ in $X_{0}$ and (5.9), for a.e. $x \in \Omega$ we get

$$
u_{j}(x)=w_{j}(x)+v_{j}(x)=\left\|w_{j}\right\|_{X_{0}, q} \frac{w_{j}(x)}{\left\|w_{j}\right\|_{X_{0}, q}}+v_{j}(x) \rightarrow \begin{cases}+\infty & \text { for a.e. } x \in\{w>0\} \\ -\infty & \text { for a.e. } x \in\{w<0\}\end{cases}
$$

as $j \rightarrow+\infty$.

Let us define the function $f_{\infty}: \Omega \rightarrow \mathbb{R}$ as

$$
f_{\infty}(x):= \begin{cases}f_{r} & \text { if } x \in\{w>0\} \\ f_{l} & \text { if } x \in\{w<0\}\end{cases}
$$

where $f_{l}$ and $f_{r}$ were introduced in (1.14). Note that $f_{\infty}$ is well defined, thanks to (5.9).

By (1.8), (5.10) and the definition of $f_{\infty}$ it follows that

$$
f\left(u_{j}(x)\right) \rightarrow f_{\infty}(x) \text { a.e. } x \in \Omega,
$$

while, by (1.9), the fact that $\Omega$ is bounded and the Lebesgue Dominated Convergence Theorem we have

$$
f\left(u_{j}\right) \rightarrow f_{\infty} \text { in } L^{\nu}(\Omega) \text { for any } \nu \in[1,+\infty)
$$

as $j \rightarrow+\infty$.

Hence, by combining (5.5) with $\varphi=w,(5.8)$ with $\varphi=v_{j}$ and (5.11), we obtain

$$
\int_{\Omega} f_{\infty}(x) w(x) d x+\int_{\Omega} h(x) w(x) d x=0,
$$

namely, writing $w(x)=w^{+}(x)-w^{-}(x)$ and taking into account the definition of $f_{\infty}$,

$$
\int_{\Omega} h(x) w(x) d x=f_{l} \int_{\Omega} w^{-}(x) d x-f_{r} \int_{\Omega} w^{+}(x) d x .
$$

This contradicts assumption (1.15). Thus, the sequence $\left\{w_{j}\right\}_{j \in \mathbb{N}}$ has to be bounded in $X_{0}$ and this concludes the proof of Proposition 12.

As a consequence of Proposition 11 (which holds true for any $\lambda \in \mathbb{R}$ ) and Proposition 12, the functional $\mathcal{J}$ has the Palais-Smale compactness property, also in the case when (1.13) occurs.

Finally, we prove that the functional $\mathcal{J}$ has the geometric feature required by the Saddle Point Theorem. As we said above, the resonance assumption affects also the proof of the particular geometric structure of the functional $\mathcal{J}$, making it more difficult than in the nonresonant setting. Indeed, here we can not use the arguments performed in the non-resonant framework, but we have to argue in a different way. For this, we will make use of (1.13) and of the Landesman-Lazer condition (1.15), which will be both crucial in the proof of the following proposition:

Proposition 13. Let $\lambda$ be as in (5.1) for some $k, m \in \mathbb{N}$, and verify (1.13). Let $K$ : $\mathbb{R}^{n} \backslash\{0\} \rightarrow(0,+\infty)$ satisfy assumptions (1.3)-(1.5). Moreover, let $f, q$ and $h$ be functions satisfying (1.8)-(1.11), (1.14) and (1.15). Then, the functional $\mathcal{J}$ verifies

$$
\inf _{u \in \mathbb{P}_{k+1}} \mathcal{J}(u)>-\infty .
$$

Proof. In order to prove Proposition 13, we argue by contradiction and assume that there exists a sequence $\left\{u_{j}\right\}_{j \in \mathbb{N}}$ in $\mathbb{P}_{k+1}$ such that

$$
\mathcal{J}\left(u_{j}\right) \rightarrow-\infty
$$

as $j \rightarrow+\infty$.

\footnotetext{
${ }^{3}$ We stress that the boundedness in $X_{0}$ imply the convergence of $v_{j}$ to some $v$ in $L^{1}\left(\mathbb{R}^{n}\right)$ and a.e. - in particular, $|v(x)| \neq+\infty$ for a.e. $x \in \Omega$.
} 
First of all, note that, by (5.1) and the orthogonality properties of $\left\{e_{1}, \ldots, e_{k}, \ldots\right\}$ (see Proposition 14-vi)), we can write $\mathbb{P}_{k+1}$ as follows

$$
\mathbb{P}_{k+1}=E_{\lambda} \oplus \mathbb{P}_{k+m+1}
$$

(recall that $\left.E_{\lambda}:=\operatorname{span}\left\{e_{k+1}, \ldots e_{k+m}\right\}\right)$.

Then, for any $j \in \mathbb{N}$ the function $u_{j}$ can be written as

$$
u_{j}=w_{j}+v_{j}
$$

with $w_{j} \in E_{\lambda}$ and $v_{j} \in \mathbb{P}_{k+m+1}$, so that $w_{j}$ and $v_{j}$ are orthogonal both in $X_{0}$ and in $L^{2}(\Omega)$, again thanks to Proposition 14-vi).

From now on we proceed by steps.

Claim 1. The following assertion holds true:

$$
\left\|w_{j}\right\|_{X_{0}, q} \rightarrow+\infty
$$

as $j \rightarrow+\infty$.

Proof. First of all, since $w_{j} \in E_{\lambda}$, note that

$$
\int_{\mathbb{R}^{n} \times \mathbb{R}^{n}}\left|w_{j}(x)-w_{j}(y)\right|^{2} K(x-y) d x d y+\int_{\Omega} q(x)\left|w_{j}(x)\right|^{2} d x=\lambda \int_{\Omega}\left|w_{j}(x)\right|^{2} d x .
$$

So, as a consequence of this, of (5.14), of the orthogonality of the $w_{j}$ and $v_{j}$, of Lemma 4 (here applied in $\mathbb{P}_{k+m+1}$ ) and of the positivity of $\lambda$, we get

$$
\begin{aligned}
\mathcal{J}\left(u_{j}\right) & =\frac{1}{2}\left\|u_{j}\right\|_{X_{0}, q}^{2}-\frac{\lambda}{2} \int_{\Omega}\left|u_{j}(x)\right|^{2} d x-\int_{\Omega} F\left(u_{j}(x)\right) d x-\int_{\Omega} h(x) u_{j}(x) d x \\
& =\frac{1}{2}\left\|w_{j}\right\|_{X_{0}, q}^{2}+\frac{1}{2}\left\|v_{j}\right\|_{X_{0}, q}^{2}-\frac{\lambda}{2}\left\|w_{j}\right\|_{L^{2}(\Omega)}^{2}-\frac{\lambda}{2}\left\|v_{j}\right\|_{L^{2}(\Omega)}^{2}-\int_{\Omega} F\left(u_{j}(x)\right) d x \\
& \geqslant \frac{1}{2}\left(1-\frac{\lambda}{\lambda_{k+m+1}}\right)\left\|v_{j}\right\|_{X_{0}, q}^{2}-\int_{\Omega} F(x) u_{j}(x) d x \\
& \left.\geqslant \frac{1}{2}\left(1-\frac{\lambda}{\lambda_{k+m+1}}\right)\left\|v_{j}\right\|_{X_{0}, q}^{2}-\tilde{C}\left\|u_{j}\right\|_{X_{0}, q}\right) d x-\int_{\Omega} h(x) u_{j}(x) d x \\
& \geqslant \frac{1}{2}\left(1-\frac{\lambda}{\lambda_{k+m+1}}\right)\left\|v_{j}\right\|_{X_{0}, q}^{2}-\tilde{C}\left\|v_{j}\right\|_{X_{0}, q}-\tilde{C}\left\|w_{j}\right\|_{X_{0}, q},
\end{aligned}
$$

also thanks to Lemma 5. So, by combining (5.13) and (5.15) we get

$$
\frac{1}{2}\left(1-\frac{\lambda}{\lambda_{k+m+1}}\right)\left\|v_{j}\right\|_{X_{0}, q}^{2}-\tilde{C}\left\|v_{j}\right\|_{X_{0}, q}-\tilde{C}\left\|w_{j}\right\|_{X_{0}, q} \rightarrow-\infty
$$

which implies necessarily that

$$
\left\|w_{j}\right\|_{X_{0}, q} \rightarrow+\infty \text { as } j \rightarrow+\infty
$$

since $\lambda=\lambda_{k+1}<\lambda_{k+m+1}$ by (5.1). Hence, Claim 1 is proved.

Now, since $E_{\lambda}$ is finite dimensional, there exists $w \in E_{\lambda}$ such that, up to a subsequence,

$$
w_{j} /\left\|w_{j}\right\|_{X_{0}, q} \rightarrow w \text { strongly in } X_{0}
$$

as $j \rightarrow+\infty$. Note that $w \not \equiv 0$, since $\|w\|=1$. Also, $w$ is an eigenfunction of problem (1.12) and so, by (1.13) $w$ is almost everywhere different from zero, say

$$
w(x) \neq 0 \text { for any } x \in \Omega \backslash \mathcal{N},
$$

where $\mathcal{N} \subset \Omega$ has zero Lebesgue measure. 
Moreover, by applying [16, Lemma 8] and [3, Theorem IV.9], up to a subsequence, we also have

$$
\begin{aligned}
& \frac{w_{j}}{\left\|w_{j}\right\|_{X_{0}, q}} \rightarrow w \quad \text { in } L^{\nu}\left(\mathbb{R}^{n}\right) \text { for any } \nu \in\left[1,2^{*}\right) \\
& \frac{w_{j}}{\left\|w_{j}\right\|_{X_{0}, q}} \rightarrow w \quad \text { a.e. in } \mathbb{R}^{n}
\end{aligned}
$$

as $j \rightarrow+\infty$. Again here and in the sequel $2^{*}$ is the exponent given in (4.1) .

Now, assume that $\left\|v_{j}\right\|_{X_{0}, q} \neq 0$ for $j$ sufficiently large. We will discuss the case when $\left\|v_{j}\right\|_{X_{0}, q}=0$ later on.

Again by applying [16, Lemma 8] and [3, Theorem IV.9] we can say that there exists $v \in X_{0}$ such that, up to a subsequence

$$
\begin{aligned}
& \frac{v_{j}}{\left\|v_{j}\right\|_{X_{0}, q}} \rightarrow v \quad \text { in } L^{\nu}\left(\mathbb{R}^{n}\right) \text { for any } \nu \in\left[1,2^{*}\right) \\
& \frac{v_{j}}{\left\|v_{j}\right\|_{X_{0}, q}} \rightarrow v \quad \text { a.e. in } \mathbb{R}^{n}
\end{aligned}
$$

as $j \rightarrow+\infty$.

Now, let us continue with some claims.

Claim 2. The following assertion holds true:

$$
\frac{\left\|w_{j}\right\|_{X_{0}, q}}{\left\|v_{j}\right\|_{X_{0}, q}} \rightarrow+\infty
$$

as $j \rightarrow+\infty$.

Proof. If $\left\|v_{j}\right\|_{X_{0}, q}$ was bounded, then Claim 2 would follow by Claim 1. Assume that $\left\|v_{j}\right\|_{X_{0}, q} \rightarrow+\infty$ as $j \rightarrow+\infty$. Writing (5.16) as follows

$$
\left\|v_{j}\right\|_{X_{0}, q}\left(\frac{1}{2}\left(1-\frac{\lambda}{\lambda_{k+m+1}}\right)\left\|v_{j}\right\|_{X_{0}, q}-\tilde{C}-\tilde{C} \frac{\left\|w_{j}\right\|_{X_{0}, q}}{\left\|v_{j}\right\|_{X_{0}, q}}\right) \rightarrow-\infty,
$$

we would get necessarily that Claim 2 holds true, by assumption (5.1). This concludes the proof of Claim 2 .

Claim 3. The following assertion holds true:

$$
\frac{F\left(u_{j}(x)\right)}{\left\|w_{j}\right\|_{X_{0}, q}} \rightarrow w(x) f_{\infty}(x) \quad \text { a.e. } x \in \Omega
$$

as $j \rightarrow+\infty$, where $f_{\infty}: \Omega \rightarrow \mathbb{R}$ is the function defined as

$$
f_{\infty}(x):= \begin{cases}f_{r} & \text { if } x \in\{w>0\} \\ f_{l} & \text { if } x \in\{w<0\}\end{cases}
$$

with $f_{l}$ and $f_{r}$ given in (1.14) and $w$ as in (5.17).

Proof. To prove this we first observe that

$$
\lim _{t \rightarrow-\infty} \frac{F(t)}{t}=f_{l} \text { and } \lim _{t \rightarrow+\infty} \frac{F(t)}{t}=f_{r} .
$$

We prove the identity for $f_{r}$, since the one for $f_{l}$ is alike. If $f_{r} \neq 0$, we can use de l'Hôpital Theorem and get (5.22). On the other hand, when $f_{r}=0$, for any $\epsilon>0$ there exists $T>0$ such that $|f(t)|<\epsilon$ for $t>T$. So, by (1.9) for $t>T$ it follows that

$$
\left|\frac{F(t)}{t}\right|=\left|\frac{1}{t}\left(\int_{0}^{T} f(\tau) d \tau+\int_{T}^{t} f(\tau) d \tau\right)\right| \leqslant M \frac{T}{t}+\epsilon \frac{(t-T)}{t} .
$$

Passing to the limit as $t \rightarrow+\infty$ and as $\epsilon \rightarrow 0$ we obtain (5.22) in this case too. 
By (5.14), Claims 1 and 2, (5.18), (5.19) and (5.20) for a.e. $x \in \Omega$ we get

$$
\begin{aligned}
u_{j}(x) & =w_{j}(x)+v_{j}(x) \\
& =\left\|w_{j}\right\|_{X_{0}, q}\left(\frac{w_{j}(x)}{\left\|w_{j}\right\|_{X_{0}, q}}+\frac{\left\|v_{j}\right\|_{X_{0}, q}}{\left\|w_{j}\right\|_{X_{0}, q}} \frac{v_{j}(x)}{\left\|v_{j}\right\|_{X_{0}, q}}\right) \\
& \rightarrow \begin{cases}+\infty & \text { for a.e. } x \in\{w>0\} \\
-\infty & \text { for a.e. } x \in\{w<0\},\end{cases}
\end{aligned}
$$

as $j \rightarrow+\infty$. In particular, fixed any $x \in \Omega$, we have that $u_{j}(x) \neq 0$ for large $j$.

Now, again by (5.14) and Claim 1, we can write

$$
\frac{F\left(u_{j}(x)\right)}{\left\|w_{j}\right\|_{X_{0}, q}}=\left(\frac{v_{j}(x)}{\left\|w_{j}\right\|_{X_{0}, q}}+\frac{w_{j}(x)}{\left\|w_{j}\right\|_{X_{0}, q}}\right) \frac{F\left(u_{j}(x)\right)}{u_{j}(x)} .
$$

By (5.22) and (5.23)

$$
\frac{F\left(u_{j}(x)\right)}{u_{j}(x)} \rightarrow \begin{cases}f_{r} & \text { for a.e. } x \in\{w>0\} \\ f_{l} & \text { for a.e. } x \in\{w<0\}\end{cases}
$$

that is

$$
\frac{F\left(u_{j}(x)\right)}{u_{j}(x)} \rightarrow f_{\infty}(x) \text { a.e } x \in \Omega
$$

as $j \rightarrow+\infty$, where $f_{\infty}$ is given in (5.21) (this function is well defined, thanks to (5.18)).

Moreover, by Claim 2 and (5.20) it follows that

$$
\frac{v_{j}(x)}{\left\|w_{j}\right\|_{X_{0}, q}}=\frac{\left\|v_{j}\right\|_{X_{0}, q}}{\left\|w_{j}\right\|_{X_{0}, q}} \frac{v_{j}(x)}{\left\|v_{j}\right\|_{X_{0}, q}} \rightarrow 0 \quad \text { a.e. } x \in \mathbb{R}^{n}
$$

as $j \rightarrow+\infty$. So, by combining (5.24)-(5.26) and by using also (5.19), we get the assertion of Claim 3.

Claim 4. The following assertion holds true:

$$
\frac{F\left(u_{j}\right)}{\left\|w_{j}\right\|_{X_{0}, q}} \rightarrow w f_{\infty} \quad \text { in } L^{1}(\Omega)
$$

as $j \rightarrow+\infty$, where $w$ is as in (5.17) and $f_{\infty}$ is defined as in (5.21).

Proof. Since $u_{j} /\left\|u_{j}\right\|_{X_{0}, q}$ is bounded in $X_{0}$, as usual by applying [16, Lemma 8] and [3, Theorem IV.9], up to a subsequence, it converges strongly in $L^{1}(\Omega)$ and there exists $\kappa \in L^{1}(\Omega)$ such that for any $j \in \mathbb{N}$

$$
\frac{\left|u_{j}(x)\right|}{\left\|u_{j}\right\|_{X_{0}, q}} \leqslant \kappa(x) \quad \text { a.e. } x \in \Omega
$$

Moreover, by the orthogonality properties of $v_{j}$ and $w_{j}$ we get

$$
\frac{\left\|u_{j}\right\|_{X_{0}, q}}{\left\|w_{j}\right\|_{X_{0}, q}}=1+\frac{\left\|v_{j}\right\|_{X_{0}, q}}{\left\|w_{j}\right\|_{X_{0}, q}}
$$

so that, by Claim 2 it follows that for any $j \in \mathbb{N}$

$$
\frac{\left\|u_{j}\right\|_{X_{0}, q}}{\left\|w_{j}\right\|_{X_{0}, q}} \leqslant C
$$

for some positive constant $C$.

As a consequence of this, (5.27) and (1.9) we get a.e. $x \in \Omega$

$$
\frac{\left|F\left(u_{j}(x)\right)\right|}{\left\|w_{j}\right\|_{X_{0}, q}} \leqslant M \frac{\left|u_{j}(x)\right|}{\left\|w_{j}\right\|_{X_{0}, q}}=M \frac{\left\|u_{j}\right\|_{X_{0}, q}}{\left\|w_{j}\right\|_{X_{0}, q}} \frac{\left|u_{j}(x)\right|}{\left\|u_{j}\right\|_{X_{0}, q}} \leqslant \bar{C} \kappa(x) \in L^{1}(\Omega)
$$


for a suitable positive constant $\bar{C}$. Then, the Lebesgue Dominated Convergence Theorem and Claim 3 yield the assertion of Claim 4 .

Claim 5. The following assertion holds true:

$$
\lim _{j \rightarrow+\infty}\left(\int_{\Omega} \frac{F\left(u_{j}(x)\right)}{\left\|w_{j}\right\|_{X_{0}, q}} d x+\int_{\Omega} h(x) \frac{u_{j}(x)}{\left\|w_{j}\right\|_{X_{0}, q}} d x\right)<0 .
$$

Proof. First of all, note that

$$
\frac{u_{j}}{\left\|w_{j}\right\|_{X_{0}, q}}=\frac{w_{j}+v_{j}}{\left\|w_{j}\right\|_{X_{0}, q}}=\frac{w_{j}}{\left\|w_{j}\right\|_{X_{0}, q}}+\frac{v_{j}}{\left\|v_{j}\right\|_{X_{0}, q}} \frac{\left\|v_{j}\right\|_{X_{0}, q}}{\left\|w_{j}\right\|_{X_{0}, q}} \rightarrow w \quad \text { in } L^{2}(\Omega),
$$

as $j \rightarrow+\infty$, thanks to (5.14), (5.19), (5.20) and Claim 2 .

As a consequence of this and by Claim 4 and (5.21) we have

$$
\begin{aligned}
\lim _{j \rightarrow+\infty}\left(\int_{\Omega} \frac{F\left(u_{j}(x)\right)}{\left\|w_{j}\right\|_{X_{0}, q}} d x\right. & \left.+\int_{\Omega} h(x) \frac{u_{j}(x)}{\left\|w_{j}\right\|_{X_{0}, q}} d x\right) \\
& =\int_{\Omega} f_{\infty}(x) w(x) d x+\int_{\Omega} h(x) w(x) d x \\
& =f_{r} \int_{\Omega} w^{+}(x) d x-f_{l} \int_{\Omega} w^{-}(x) d x+\int_{\Omega} h(x) w(x) d x<0
\end{aligned}
$$

since (1.15) holds true. This ends the proof of Claim 5 .

Now, we can conclude the proof of Proposition 13. Indeed, arguing as (5.15) and using (5.1), we get

$$
\begin{aligned}
\mathcal{J}\left(u_{j}\right) & \geqslant \frac{1}{2}\left(1-\frac{\lambda}{\lambda_{k+m+1}}\right)\left\|v_{j}\right\|_{X_{0}, q}^{2}-\int_{\Omega} F\left(u_{j}(x)\right) d x-\int_{\Omega} h(x) u_{j}(x) d x \\
& \geqslant-\left\|w_{j}\right\|_{X_{0}, q}\left(\int_{\Omega} \frac{F\left(u_{j}(x)\right)}{\left\|w_{j}\right\|_{X_{0}, q}} d x+\int_{\Omega} h(x) \frac{u_{j}(x)}{\left\|w_{j}\right\|_{X_{0}, q}} d x\right)
\end{aligned}
$$

so that, by Claim 1 and Claim 5, we deduce

$$
\mathcal{J}\left(u_{j}\right) \rightarrow+\infty \quad \text { as } j \rightarrow+\infty,
$$

which contradicts (5.13). Hence, Proposition 13 holds true in the case when $\left\|v_{j}\right\|_{X_{0}, q} \neq 0$ for $j$ large enough.

Finally, it remains to consider the case when $\left\|v_{j}\right\|_{X_{0}, q}=0$ for $j$ sufficiently large (up to a subsequence). In this setting, using the same arguments as above, the proof can be repeated in a simpler way. For the sake of clarity and for reader's convenience we prefer to give full details.

Since $\left\|v_{j}\right\|_{X_{0}, q}=0$ for $j$ sufficiently large, it easily follows that

$$
v_{j} \rightarrow 0 \text { in } X_{0}
$$

as $j \rightarrow+\infty$. Hence, by [16, Lemma 8] and [3, Theorem IV.9] up to a subsequence

$$
\begin{array}{ll}
v_{j} \rightarrow 0 & \text { in } L^{\nu}\left(\mathbb{R}^{n}\right) \text { for any } \nu \in\left[1,2^{*}\right) \\
v_{j} \rightarrow 0 & \text { a.e. in } \mathbb{R}^{n}
\end{array}
$$

as $j \rightarrow+\infty$.

As a consequence of this and by (5.14), (5.19) and Claim 1, we get that

$$
\frac{u_{j}}{\left\|w_{j}\right\|_{X_{0}, q}}=\frac{w_{j}}{\left\|w_{j}\right\|_{X_{0}, q}}+\frac{v_{j}}{\left\|w_{j}\right\|_{X_{0}, q}} \rightarrow w \text { in } L^{\nu}\left(\mathbb{R}^{n}\right) \text { for any } \nu \in\left[1,2^{*}\right),
$$

so that

$$
\frac{u_{j}(x)}{\left\|w_{j}\right\|_{X_{0}, q}} \rightarrow w(x) \text { a.e } x \in \Omega
$$


as $j \rightarrow+\infty$, and, for any $j \in \mathbb{N}$ and a.e. $x \in \Omega$

$$
\frac{\left|u_{j}(x)\right|}{\left\|w_{j}\right\|_{X_{0}, q}} \leqslant \kappa_{\nu}(x)
$$

for some $\kappa_{\nu} \in L^{\nu}(\Omega)$.

Also, again by (5.14), Claim 1, (5.19) and (5.30), we deduce that a.e. $x \in \Omega$

$$
u_{j}(x)=w_{j}(x)+v_{j}(x)=\left\|w_{j}\right\|_{X_{0}, q} \frac{w_{j}(x)}{\left\|w_{j}\right\|_{X_{0}, q}}+v_{j}(x) \rightarrow\left\{\begin{array}{cc}
+\infty & \text { if } x \in\{w>0\} \\
-\infty & \text { if } x \in\{w<0\}
\end{array}\right.
$$

as $j \rightarrow+\infty$, thanks to (5.18).

Hence, by (5.22) and (5.33), also in this case we get

$$
\frac{F\left(u_{j}(x)\right)}{u_{j}(x)} \rightarrow f_{\infty}(x) \text { a.e. } x \in \Omega
$$

as $j \rightarrow+\infty$, where $f_{\infty}$ is the function defined in (5.21).

Now, we have that

$$
\frac{F\left(u_{j}(x)\right)}{\left\|w_{j}\right\|_{X_{0}, q}}=\left(\frac{v_{j}(x)}{\left\|w_{j}\right\|_{X_{0}, q}}+\frac{w_{j}(x)}{\left\|w_{j}\right\|_{X_{0}, q}}\right) \frac{F\left(u_{j}(x)\right)}{u_{j}(x)} \rightarrow w(x) f_{\infty}(x) \text { a.e. } x \in \Omega,
$$

as $j \rightarrow+\infty$, thanks to (5.14), (5.19), (5.30) and (5.34).

Furthermore, by (1.9) and (5.32) we get that a.e. $x \in \Omega$ and for any $j \in \mathbb{N}$

$$
\frac{\left|F\left(u_{j}(x)\right)\right|}{\left\|w_{j}\right\|_{X_{0}, q}} \leqslant M \frac{\left|u_{j}(x)\right|}{\left\|w_{j}\right\|_{X_{0}, q}} \leqslant M \kappa_{1}(x) \in L^{1}(\Omega),
$$

so that, using also (5.35), we obtain

$$
\frac{F\left(u_{j}\right)}{\left\|w_{j}\right\|_{X_{0}, q}} \rightarrow w f_{\infty} \text { in } L^{1}(\Omega)
$$

as $j \rightarrow+\infty$.

Now, with (1.15), (5.31) (here used with $\nu=2<2^{*}$ ) and (5.36), arguing as in Claim 5, we can show that

$$
\lim _{j \rightarrow+\infty}\left(\int_{\Omega} \frac{F\left(u_{j}(x)\right)}{\left\|w_{j}\right\|_{X_{0}, q}} d x+\int_{\Omega} h(x) \frac{u_{j}(x)}{\left\|w_{j}\right\|_{X_{0}, q}} d x\right)<0 .
$$

Thus, the conclusion of Proposition 13 follows as in the previous case. This ends the proof of Proposition 13 .

Finally, we are ready to prove Theorem 1, in the resonant case.

5.1. Proof of Theorem 1 in the resonant setting. First of all, let us check the geometric structure of the functional $\mathcal{J}$. For this, let

$$
I=\inf _{u \in \mathbb{P}_{k+1}} \mathcal{J}(u) .
$$

By Proposition 13 and the fact that $\mathcal{J} \not \equiv+\infty$, we have that $I \in \mathbb{R}$. Moreover, by Lemma 7 , there exists $R>0$ such that for any $u \in \mathbb{H}_{k}$ with $\|u\|_{X_{0}, q} \geqslant R$ it holds true that

$$
\mathcal{J}(u)<-|I| \leqslant I \text {. }
$$

Then, as a consequence of this, we get

$$
\sup _{\substack{u \in \mathbb{H}_{k} \\\|u\|_{X_{0}, q}=R}} \mathcal{J}(u) \leqslant \sup _{\substack{u \in \mathbb{H}_{k} \\\|u\|_{X_{0}, q} \geqslant R}} \mathcal{J}(u)<I=\inf _{u \in \mathbb{P}_{k+1}} \mathcal{J}(u),
$$

that is $\mathcal{J}$ has the geometry required by the Saddle Point Theorem (see [12, Theorem 4.6]).

Finally, by Proposition 11 (which holds true for any $\lambda \in \mathbb{R}$ ) and Proposition 12, the functional $\mathcal{J}$ satisfies the Palais-Smale condition. 
Hence, we can make use of the Saddle Point Theorem in order to obtain a critical point $u \in X_{0}$ of $\mathcal{J}$. This concludes the proof of Theorem 1 in the resonant case.

\section{Appendix A. An eigenvalue PROBlem For $-\mathcal{L}_{K}+q$}

This appendix is devoted to the study of the eigenvalue problem (1.12). More precisely, we study the weak formulation of (1.12), which consists in the following problem

$$
\left\{\begin{array}{c}
\int_{\mathbb{R}^{n} \times \mathbb{R}^{n}}(u(x)-u(y))(\varphi(x)-\varphi(y)) K(x-y) d x d y+\int_{\Omega} q(x) u(x) \varphi(x) d x \\
=\lambda \int_{\Omega} u(x) \varphi(x) d x \quad \forall \varphi \in X_{0} \\
u \in X_{0} .
\end{array}\right.
$$

We recall that $\lambda \in \mathbb{R}$ is an eigenvalue of $-\mathcal{L}_{K}+q$ provided there exists a non-trivial solution $u \in X_{0}$ of problem (A.1) and, in this case, any solution will be called an eigenfunction corresponding to the eigenvalue $\lambda$.

For the proof of the next result we refer to [17, Proposition 9 and Appendix A], where the case when $q \equiv 0$ was considered. The proof of [17, Proposition 9] can be easily adapted in order to get the following result:

Proposition 14. Let $s \in(0,1), n>2 s, \Omega$ be an open, bounded subset of $\mathbb{R}^{n}$ and let $K: \mathbb{R}^{n} \backslash\{0\} \rightarrow(0,+\infty)$ be a function satisfying assumptions (1.3)-(1.5). Moreover, let $q: \Omega \rightarrow \mathbb{R}$ be a function verifying (1.10). Then,

(i) problem (A.1) admits an eigenvalue $\lambda_{1}$ which is positive and that can be characterized as follows

$$
\lambda_{1}=\min _{\substack{u \in X_{0} \\\|u\|_{L^{2}(\Omega)}=1}}\left(\int_{\mathbb{R}^{n} \times \mathbb{R}^{n}}|u(x)-u(y)|^{2} K(x-y) d x d y+\int_{\Omega} q(x)|u(x)|^{2} d x\right),
$$

or, equivalently,

$$
\lambda_{1}=\min _{u \in X_{0} \backslash\{0\}} \frac{\int_{\mathbb{R}^{n} \times \mathbb{R}^{n}}|u(x)-u(y)|^{2} K(x-y) d x d y+\int_{\Omega} q(x)|u(x)|^{2} d x}{\int_{\Omega}|u(x)|^{2} d x} ;
$$

(ii) there exists a non-negative function $e_{1} \in X_{0}$, which is an eigenfunction corresponding to $\lambda_{1}$, attaining the minimum in (A.2), that is $\left\|e_{1}\right\|_{L^{2}(\Omega)}=1$ and

$$
\lambda_{1}=\int_{\mathbb{R}^{n} \times \mathbb{R}^{n}}\left|e_{1}(x)-e_{1}(y)\right|^{2} K(x-y) d x d y+\int_{\Omega} q(x)\left|e_{1}(x)\right|^{2} d x ;
$$

(iii) $\lambda_{1}$ is simple, that is if $u \in X_{0}$ is a solution of the following equation

$$
\begin{aligned}
\int_{\mathbb{R}^{n} \times \mathbb{R}^{n}}(u(x)-u(y))(\varphi(x)-\varphi(y)) & K(x-y) d x d y+\int_{\Omega} q(x)|u(x)|^{2} d x \\
& =\lambda_{1} \int_{\Omega} u(x) \varphi(x) d x \quad \forall \varphi \in X_{0},
\end{aligned}
$$

then $u=\zeta e_{1}$, with $\zeta \in \mathbb{R}$;

(iv) the set of the eigenvalues of problem (A.1) consists of a sequence $\left\{\lambda_{k}\right\}_{k \in \mathbb{N}}$ with

$$
0<\lambda_{1}<\lambda_{2} \leqslant \ldots \leqslant \lambda_{k} \leqslant \lambda_{k+1} \leqslant \ldots
$$

and

$$
\lambda_{k} \rightarrow+\infty \text { as } k \rightarrow+\infty .
$$

Moreover, for $k \in \mathbb{N}$ the eigenvalues can be characterized as follows:

$$
\lambda_{k+1}=\min _{\substack{u \in \mathbb{P}_{k+1} \\\|u\|_{L^{2}(\Omega)}=1}}\left(\int_{\mathbb{R}^{n} \times \mathbb{R}^{n}}|u(x)-u(y)|^{2} K(x-y) d x d y+\int_{\Omega} q(x)|u(x)|^{2} d x\right),
$$


or, equivalently,

$$
\lambda_{k+1}=\min _{u \in \mathbb{P}_{k+1} \backslash\{0\}} \frac{\int_{\mathbb{R}^{n} \times \mathbb{R}^{n}}|u(x)-u(y)|^{2} K(x-y) d x d y+\int_{\Omega} q(x)|u(x)|^{2} d x}{\int_{\Omega}|u(x)|^{2} d x},
$$

where

$$
\mathbb{P}_{k+1}:=\left\{u \in X_{0}:\left\langle u, e_{j}\right\rangle_{X_{0}, q}=0 \quad \forall j=1, \ldots, k\right\} ;
$$

(v) for any $k \in \mathbb{N}$ there exists a function $e_{k+1} \in \mathbb{P}_{k+1}$, which is an eigenfunction corresponding to $\lambda_{k+1}$, attaining the minimum in (A.3), that is $\left\|e_{k+1}\right\|_{L^{2}(\Omega)}=1$ and

$$
\lambda_{k+1}=\int_{\mathbb{R}^{n} \times \mathbb{R}^{n}}\left|e_{k+1}(x)-e_{k+1}(y)\right|^{2} K(x-y) d x d y+\int_{\Omega} q(x)\left|e_{k+1}(x)\right|^{2} d x ;
$$

(vi) the sequence $\left\{e_{k}\right\}_{k \in \mathbb{N}}$ of eigenfunctions corresponding to $\lambda_{k}$ is an orthonormal basis of $L^{2}(\Omega)$ and an orthogonal basis of $X_{0}$;

(vii) each eigenvalue $\lambda_{k}$ has finite multiplicity; more precisely, if $\lambda_{k}$ is such that

$$
\lambda_{k-1}<\lambda_{k}=\ldots=\lambda_{k+h}<\lambda_{k+h+1}
$$

for some $h \in \mathbb{N}_{0}$, then the set of all the eigenfunctions corresponding to $\lambda_{k}$ agrees with

$$
\operatorname{span}\left\{e_{k}, \ldots, e_{k+h}\right\} .
$$

For further properties of the eigenvalues and the eigenfunctions of the fractional Laplace operator (and, in general, of non-local integrodifferential operators) we refer to [13, Proposition 5], [19, Proposition 4] and [21].

\section{REFERENCES}

[1] M. Badiale and E. Serra, Semilinear Elliptic Equations for Beginners, Springer, Berlin (2011).

[2] B. Barrios, E. Colorado, A. De Pablo and U. Sanchez, On some critical problems for the fractional Laplacian operator, J. Differential Equations 252, no. 11, 6133-6162 (2012).

[3] H. BrÉzis, Analyse fonctionelle. Théorie et applications, Masson, Paris (1983).

[4] X. CABré And J. TAN, Positive solutions of nonlinear problems involving the square root of the Laplacian, Adv. Math. 224, no. 5, 2052-2093 (2010).

[5] A. CAPElla, Solutions of a pure critical exponent problem involving the half-Laplacian in annularshaped domains, Commun. Pure Appl. Anal. 10, no. 6, 1645-1662 (2011).

[6] D.G. De Figueiredo And J.P. Gossez, Strict monotonicity of eigenvalues and unique continuation, Comm. Partial Differential Equations 17, no. 1-2, 339-346 (1992).

[7] E. Di Nezza, G. Palatucci And E. Valdinoci, Hitchhiker's guide to the fractional Sobolev spaces, Bull. Sci. Math. 136, no. 5, 521-573 (2012).

[8] A. Fiscella, Saddle point solutions for non-local elliptic operators, preprint (2012).

[9] A. Fiscella, R. Servadei And E. Valdinoci, A resonance problem for non-local elliptic operators, preprint, available at http://www.ma.utexas.edu/mp_arc-bin/mpa?yn=12-61 .

[10] D. JERISON AND C.E. KENIG, Unique continuation and absence of positive eigenvalues for Schrödinger operators, Ann. of Math. (2) 121, no. 3, 463-494 (1985).

[11] E.M. LANDESMAN AND A.C. LAZER, Nonlinear perturbations of linear elliptic boundary value problems at resonance, J. Math. Mech. 19, 609-623 (1969/1970).

[12] P.H. Rabinowitz, Minimax methods in critical point theory with applications to differential equations, CBMS Reg. Conf. Ser. Math., 65, American Mathematical Society, Providence, RI (1986).

[13] R. SERVADEI, The Yamabe equation in a non-local setting, preprint, available at http://www . ma.utexas .edu/mp_arc-bin/mpa?yn=12-40.

[14] R. SERvadei, A critical fractional Laplace equation in the resonant case, to appear in Topol. Methods Nonlinear Anal.

[15] R. Servadei AND E. VAldinOci, Lewy-Stampacchia type estimates for variational inequalities driven by (non) local operators, to appear in Rev. Mat. Iberoam.

[16] R. Servadei And E. Valdinoci, Mountain Pass solutions for non-local elliptic operators, J. Math. Anal. Appl., 389, 887-898 (2012).

[17] R. Servadei And E. VAldinoci, Variational methods for non-local operators of elliptic type, to appear in Discrete Contin. Dyn. Systems. 
[18] R. Servadei And E. VAldinoci, The Brezis-Nirenberg result for the fractional Laplacian, to appear in Trans. AMS.

[19] R. Servadei And E. VAldinoci, A Brezis-Nirenberg result for non-local critical equations in low dimension, preprint, available at http://www.ma.utexas.edu/mp_arc-bin/mpa?yn=12-41 .

[20] R. Servadei And E. VAldinoci, Fractional Laplacian equations with critical Sobolev exponent, preprint, available at http://www.math.utexas.edu/mp_arc-bin/mpa?yn=12-58 .

[21] R. Servadei And E. VAldinoci, Weak and viscosity solutions of the fractional Laplace equation, preprint, available at http://www.ma.utexas.edu/mp_arc-bin/mpa?yn=12-82 .

[22] J. TAN, The Brezis-Nirenberg type problem involving the square root of the Laplacian, Calc. Var. Partial Differential Equations, 36, no. 1-2, 21-41 (2011). ITALY

Dipartimento di Matematica, Università di Milano, Via Cesare Saldini 50, 20133 Milano,

E-mail address: alessio.fiscella@unimi.it

Dipartimento di Matematica, Università della Calabria, Ponte Pietro Bucci 31 B, 87036 Arcavacata di Rende (Cosenza), Italy

E-mail address: servadei@mat.unical.it

Dipartimento di Matematica, Università di Milano, Via Cesare Saldini 50, 20133 Milano, ITALY

Istituto di Matematica Applicata e Tecnologie Informatiche, Consiglio Nazionale delle Ricerche, Via Ferrata 1, 27100 Pavia, Italy

E-mail address: valdinoci@mat.uniroma2.it 